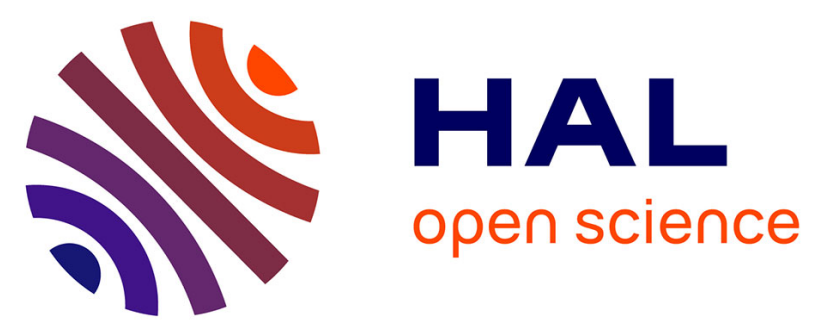

\title{
Ion slowing down and charge exchange at small impact parameters selected by channeling: superdensity effects
}

A. L'Hoir, A. Adoui, F. Barrué, A. Billebaud, F. Bosch, A. Bräuning-Demian, H. Bräuning, A. Cassimi, M. Chevallier, C. Cohen, et al.

\section{- To cite this version:}

A. L'Hoir, A. Adoui, F. Barrué, A. Billebaud, F. Bosch, et al.. Ion slowing down and charge exchange at small impact parameters selected by channeling: superdensity effects. 6th International Symposium on Swift Heavy Ions in Matter SHIM 2005, May 2005, Aschaffenburg, Germany. pp.1-14, 10.1016/j.nimb.2005.11.055 . in2p3-00025220

HAL Id: in2p3-00025220

https://hal.in2p3.fr/in2p3-00025220

Submitted on 13 Dec 2005

HAL is a multi-disciplinary open access archive for the deposit and dissemination of scientific research documents, whether they are published or not. The documents may come from teaching and research institutions in France or abroad, or from public or private research centers.
L'archive ouverte pluridisciplinaire HAL, est destinée au dépôt et à la diffusion de documents scientifiques de niveau recherche, publiés ou non, émanant des établissements d'enseignement et de recherche français ou étrangers, des laboratoires publics ou privés. 


\title{
Ion slowing down and charge exchange at small impact parameters selected by channeling: superdensity effects.
}
A. L'Hoir ${ }^{(1)}$
A. $\operatorname{Adoui}^{(2)}$
F. Barrué(3)
A. Billebaud ${ }^{(4)}$
F. Bosch ${ }^{(5)}$
A. Bräuning-Demian ${ }^{(5)}$
H. Bräuning ${ }^{(6)}$
A. Cassimi $(2$
M. Chevallier (3)
C. Cohen ${ }^{(1)}$
D. Dauvergne ${ }^{(3)}$
C.E. Demonchy ${ }^{(7)}$
L. Giot ${ }^{(7)}$
R. Kirsch
A. Gumberidze ${ }^{(5)}$
C. Kozhuharov ${ }^{(5)}$
D. Liesen ${ }^{(5)}$
W. $\operatorname{Mittig}^{(7)}$
P.H. Mokler ${ }^{(5)}$
S. Pita ${ }^{(7)}$
J.C. Poizat ${ }^{(3)}$
C. $\operatorname{Ray}^{(3)}$
P. Roussel-Chomaz ${ }^{(7)}$
H. Rothard ${ }^{(2)}$
J.P. Rozet ${ }^{(1)}$
Th. Stöhlker ${ }^{(2)}$
M. Tarisien ${ }^{(2)}$
E. Testa ${ }^{(3)}$
S. Toleikis ${ }^{(2)}$
M. Toulemonde ${ }^{(2)}$
D. Vernhet ${ }^{(1)}$

July 13, 2005

\begin{abstract}
In two experiments performed with $20-30 \mathrm{MeV} / \mathrm{u}$ highly charged heavy ions $\left(\mathrm{Pb}^{56+}, \mathrm{U}^{91+}\right)$ channeled through thin silicon crystals, we observed the original features of superdensity, associated to the glancing collisions with atomic rows undergone by part of the incident projectiles. In particular the very high collision rate yields a quite specific charge exchange regime, that leads to a higher ionization probability than in random conditions. X-ray measurements show that electrons captured in outershells are prevented from being stabilized, which enhances the lifetime of the projectile innershell vacancies. The charge state distributions and the energy loss spectra are compared to Monte-Carlo simulations. These simulations confirm, extend and illustrate the qualitative analysis of the experimental results.
\end{abstract}

PACS: 61.85. +p; 34.70. +e

Keywords: channeling; energy loss; charge exchange; heavy ions, NII; EII; REC; MEC; impact parameter.

corresponding author: lhoir@gps.jussieu.fr

(1) Institut de Nanosciences de Paris, CNRS UMR 7588, Universités Paris 6 et Paris 7, 2 Place Jussieu, 75251 Paris Cedex 05, France 
${ }^{(2)}$ Centre Interdisciplinaire de Recherche avec les Ions Lourds, UMR 11 CNRS-CEA, rue Claude Bloch, 14040 Caen Cedex, France

(3) Institut de Physique Nucléaire de Lyon and IN2P3, Université Claude Bernard Lyon-I, 4 rue Enrico Fermi, 69622 Villeurbanne Cedex, France

(4) LPSC, 38026 Grenoble Cedex, France

(5) Gesellschaft für Schwerionenforschung mbH Darmstadt, Planckstrasse 1, D-64291 Darmstadt, Germany

(6) Universität Giessen, Heinrich-Buff-Ring 16, D-35392 Giessen, Germany

(7) GANIL, BP 5027, 14076 Caen Cedex 5, France 


\section{Introduction}

Channeling of swift heavy ions in crystals is a powerful tool to study particle-solid interactions. In particular one can study interactions with valence or conduction electrons as channeling conditions restrict the accessible transverse space of projectiles according to their transverse energy. Most experimental channeling studies have dealt with the best channeled projectiles that see the crystal as a quasi-free electron gas target. One can also use the relation between the transverse energy $E_{\perp}$ and the available transverse space to study how processes as energy loss, secondary electron emission or charge exchange depend on the projectile impact parameter with respect to atomic rows $[1,2,3,4]$. A particular group of projectiles may deserve attention, that are the projectiles with a transverse energy close to the critical transverse energy $E_{\perp c}$. For an incident beam aligned along an axial or planar direction, these "critical" projectiles enter the crystal with an impact parameter (distance to the row) close to the thermal vibration amplitude. After their first glancing collision in the entrance surface region, critical projectiles are deflected by an angle nearly equal to the so-called channeling critical angle $\Psi_{c}$ and may suffer further glancing collisions on neighboring atomic rows or planes. This group of projectiles is the most abundant when the incident beam and the crystal axis or plane make an angle close to $\Psi_{c}$. Those high transverse energy projectiles are the main subject of this paper. In what follows, we will mainly focus on energy loss and charge exchange processes.

Critical channeling has been known for a long time for increasing the rate of energy loss above the "normal" value measured in random conditions. One of the challenges in material science is to understand the relation between the energy deposited in solids by inelastic processes and the creation of defects, in particular the formation of tracks. For this, experimentalists have tried to find conditions where the density of energy deposition by a projectile is maximized. One way is to use a cluster projectile for which energy deposition (before the constituents scatter away) is roughly multiplied by the number of atoms in the cluster [5]. Another way, as shown by Vickridge et al. [6], could be to use projectiles in critical channeling conditions. Using a narrow nuclear resonance the authors showed that $1 \mathrm{MeV}$ protons entering close to [110] atomic rows of an aluminum single crystal have, during their first glancing collision, an energy loss rate nearly one order of magnitude larger than in random conditions. Applied to the case of heavy ions, this method could allow to reach locally enormous linear densities of energy deposition, above $100 \mathrm{keV} / \mathrm{nm}$. It must be noted that the energy deposition by a charged projectile is much more localized (at most a few nanometers around the ion track) than in the case of intense short-pulsed lasers. 
We present here new features observed in the energy loss and charge exchange of fast heavy ions in critical axial channeling through a thin crystal. We describe and analyze (in particular with the help of a Monte-Carlo code) two channeling experiments with thin Si crystals in which we observe energy losses and charge distributions of transmitted heavy ions, secondary electron and/or X-ray emissions. The fingerprints of critical channeling are clearly visible on each of these processes. In particular charge distributions reveal a new effect of superdensity. It results from a combination of two factors: $i$ ) the impact parameter dependence of the interactions governing charge exchange for high transverse energy ions (non radiative capture also called mechanical capture, MEC, ionization and excitation by impact on the target nuclei, respectively NII and NIE) and ii) the high rate of collisions undergone in atomic rows by critical projectiles (we use here the word rate instead of frequency to differentiate this effect from the well known coherent resonant excitation of atomic levels of channeled ions $[3,7])$.

The effect of the collision rate has been observed [8] since long when comparing equilibrium charge state distributions obtained after crossing gas or dense solid targets. It results in a higher mean charge state in the case of dense targets. The close collisions of the projectile with target atoms may promote electrons to excited states. According to the interpretation of Bohr-Lindhard, whereas in a gas de-excitation takes place before the occurrence of the next collision, in a solid the collision rate may be too high for de-excitation to happen, and then excited electrons have an enhanced probability to be lost. In fact, we are studying in this paper a "superdensity effect" related to the fact that the collision rate of critical projectiles may exceed in part of their trajectories that of "random" projectiles by more than one order of magnitude. We will also show how critical projectiles could be selected and used in specific applications in material science.

\section{Ion channeling}

Ion channeling is a well documented subject (see [9] and references therein). In this paragraph, we insist on peculiar aspects of channeling, concerning mainly the behavior of particles with high transverse energy.

\subsection{Binary collisions versus transverse potential description.}

The ion trajectory in a crystal is essentially governed by elastic collisions on the screened target nuclei. For a beam with a direction parallel or nearly parallel to a major crystallographic direction, one generally defines a transverse energy $E_{\perp}$ associated to the motion 
of the ions in the transverse plane, perpendicular to the crystallographic direction as:

$$
E_{\perp}=U\left(\mathbf{r}_{\perp}\right)+E \varphi^{2}
$$

where $E \varphi^{2}$ is a transverse kinetic energy ( $E$ is the kinetic energy of the ion, $\varphi$ is the angle between the ion velocity direction and the crystallographic direction $z$ considered), and $U\left(\mathbf{r}_{\perp}\right)$ a transverse potential energy defined as the 3D interaction potential $U(\mathbf{r})$ between the ion and the crystal, averaged along the $z$ direction. Such a transverse potential is represented in figure1- $a$ in the case of the [110] axis of silicon (diamond structure) per unit ion charge. When one needs to calculate the trajectories of ions channeled in a crystal, two very different approaches may be used: i) the trajectories may be calculated using the transverse potential $U\left(\mathbf{r}_{\perp}\right)$ and $\left.i i\right)$ the motion of the ion may be considered as a succession of binary collisions with neighboring target atoms. The latter approach is the one used in Monte-Carlo calculations. This is a fully justified approach for ions with high $E_{\perp}$ that may induce small impact parameter collisions with the vibrating target atoms. In the first approach on the contrary, the ion is assumed to move in a static potential averaged over $z$, a description that reflects neither the discrete series of binary collisions, nor the thermal vibration of the target atoms. This approach may hence be used for rather large impact parameters $b$ at the scale of the thermal vibration amplitude (typically, $b \gtrsim 0.2 \AA$ ). It implies the conservation of $E_{\perp}$ for each particle. In fact, $E_{\perp}$ increases with depth $z$ (in connection with angular multiple scattering on target electrons, and for large $E_{\perp}$ ions, with a random scattering component induced by the displacement of target nuclei from regular lattice sites, i.e. by thermal vibrations), and the description may be improved by introducing an appropriate $d E_{\perp} / d z$ function. Considering the aim of this paper mainly devoted to projectiles with large $E_{\perp}$, the continuum approach is inappropriate and the Monte-Carlo approach will be used.

\subsection{Critical trajectories and critical angle}

When the incident beam is parallel to a given crystallographic direction, for a given ion, the transverse energy at the crystal surface may be written $E_{\perp \text { in }}=U\left(\mathbf{r}_{\perp \text { in }}\right)$ (zero entrance angle $\varphi_{i n}$ ). For small $r_{\perp \text { in }}$ (particle entering the crystal close to a string or a plane) the particle is strongly repelled from the string (plane).

After some penetration depth $z_{1}$, the ions are far from the atomic rows, the transverse potential reaches a minimum value $(\approx 0)$ and the trajectory gets an angle $\varphi_{1}$ given by $E_{\perp i n} \simeq E \varphi_{1}^{2}$. This lead to an oscillating trajectory, with a succession of close interactions with axes (planes) separated by large sections of the trajectory corresponding to large 
impact parameter collisions. Close to the rows (planes), collisions occur at a very high rate (the interatomic distance on the [110] axis of silicon is $a_{S i} / \sqrt{2}=3.84 \AA$, where $a_{S i}=5.43 \AA$ is the parameter of the cubic structure of silicon, and for $20 \mathrm{MeV} / \mathrm{u}$ ions, the time interval between collisions is $6.2 \times 10^{-18} \mathrm{~s}$ ), and are correlated (slowly varying impact parameters). An example of ion trajectories is given in figure 2- $a$ for $29 \mathrm{MeV} / \mathrm{u}$ $\mathrm{Pb}$ ions channeled along the [110] axis of silicon.

When $r_{\perp i n}$ is close to the root mean square amplitude $\rho$ of the thermal vibrations, $\varphi_{1}$ reaches a value $\Psi_{c}$ given by $E_{\perp i n}=E_{\perp c}=U(\rho)=E \Psi_{c}^{2}$. We shall consider that $\Psi_{c}$ is a critical angle for channeling: for $E_{\perp i n} \gtrsim E_{\perp c}$ the transverse energy conservation is no more valid and on the contrary $E_{\perp}$ increases rapidly, the higher $E_{\perp}$, the faster the increase. For an aligned beam, particles with $E_{\perp \text { in }} \gtrsim E_{\perp c}$ represent a small fraction of the beam (a few percent). These high $E_{\perp}$ ions are critical particles (depending on the process studied, the proportion of critical particles may exceed a few percent).

If the beam is tilted by a given angle $\varphi_{i n}$, the transverse energy writes $E_{\perp i n}=U\left(\mathbf{r}_{\perp i n}\right)+$ $E \varphi_{i n}^{2}$. If $E \varphi_{i n}^{2} \gtrsim E_{\perp c}$, all the particles of the beam may approach the strings (planes) at distances $r_{\perp \text { in }} \lesssim \rho$, and most of them are hence critical particles. However, as shown in figure 2- $b$, the behavior of critical particles corresponds in no way to a "random" situation i.e. for which ions sample a random medium. On the contrary, correlated collisions with strings (planes) still exist and are particularly efficient for processes such as energy loss and charge exchange. The experiments reported in this paper were mainly devoted to the study of those critical particles, and in particular, to peculiar aspects of the charge exchanges they experience.

Using a simple screened atomic potential, Lindhard [10] has obtained a good estimate of $\Psi_{c}$ for axial channeling of swift ions, which evidences simple scaling laws:

$$
\Psi_{c}=\sqrt{\frac{2 Z_{i o n} Z_{t} e^{2}}{E d}}
$$

where $Z_{i o n}$ and $Z_{t}$ are respectively the particle and target atomic numbers, $d$ the interatomic distance in the row and $e$ the elementary charge. For $20 \mathrm{MeV} / \mathrm{u}$ bare uranium ions in [110] silicon, $\Psi_{c}=1.4 \mathrm{mrad}$.

\subsection{Fitting the data}

In order to fit the experimental data that are presented in the next sections, the trajectories, energy loss and charge exchange of ions in silicon were calculated by using Monte-Carlo simulations. The principles of these calculations and the approximation 
used are described in appendix A. These simulations provide the energy loss and charge exchange experienced by an ion along its trajectory.

In what concerns energy loss, the transfers to the valence gas and to core target electrons are treated separately. For the latter, we used the very rough local density approximation (LDA) in order to provide an impact parameter dependence of energy transfers [11]. A comparison of our experimental results with simulations using a more refined stopping theory (Binary theory of electronic stopping [12]) will be undertaken in the near future.

In order to describe the charge exchange processes, projectile ionization by EII and NII, excitation by the target nuclei and electrons, electron capture by MEC and REC and cascade of the excited electrons are considered in the Monte-Carlo code. The values of all these cross sections are not known for all $n$ values. For this reason we restrict our description to only 5 shells. This may be a crude approximation. It may be justified if capture and loss of electrons in $(n>5)$-shells cancel each other and do not influence too much the population of the $(n \leq 5)$-shells.

\section{Experimental conditions}

The two experiments presented here were performed with fast heavy ion beams aligned with the [110] axial direction of a thin silicon crystal. In both cases the incident species had a charge state far from equilibrium in random conditions at its velocity and the crystal was thin enough to allow a large fraction of the projectiles incident in alignment conditions to be transmitted frozen in their initial charge state.

In the first experiment performed at GANIL (Caen) $29 \mathrm{MeV} / \mathrm{u} \mathrm{Pb}^{56+}$ ions were sent, through the beam line SPEG, onto a silicon crystal of effective thickness $1.1 \mu \mathrm{m}$. In the second experiment, performed at GSI (Darmstadt) $20 \mathrm{MeV} / \mathrm{u}$ H-like $U^{91+}$ ions were extracted from the ESR storage ring, after cooling and deceleration, to be sent, through the beam line of cave A, onto a silicon crystal of effective thickness $11.7 \mu \mathrm{m}$. Some features were common to the two experiments :

i) X-rays were detected by a Ge-detector viewing the crystal at $90^{\circ}$ to the beam direction;

ii) the crystal was brought to a positive potential (about $10 \mathrm{kV}$ ) that allowed us to collect low energy electrons, emitted from both surfaces of the crystal, in two surface barrier detectors, and then to measure the multiplicity of backward and forward emission associated to the passage of each individual projectile through the crystal; 
iii) charge state and energy distributions were analyzed by a magnetic spectrometer. Experimental details can be found in [13] and [4] for the GSI and GANIL experiments respectively.

\section{Channeling experiment with $29 \mathrm{MeV} / \mathrm{u} \mathrm{Pb}^{56+}$}

\subsection{Charge state distributions}

\subsubsection{Experimental results}

In this experiment, $29 \mathrm{MeV} / \mathrm{u} \mathrm{Pb}^{56+}$ ions enter the crystal with $K$ and $L$-shells completely filled and with 16 electrons on M-shell: the dominant process is M-shell ionization. In figure 3 we show various charge state distributions $F\left(Q_{\text {out }}\right)$ for ions transmitted through a $1.1 \mu \mathrm{m} \mathrm{Si}$ crystal in various conditions. The Gauss-shaped distribution $F_{R}\left(Q_{\text {out }}\right)$ obtained for a random crystal orientation is centered around a mean charge of 68.5. We also show the "equilibrated" distribution calculated with the semi-empirical formula proposed by Leon et al. [14], that is centered around a mean charge of 71.5 and that indicates that charge equilibrium is not reached in the thin $1.1 \mu \mathrm{m}$ silicon target, even for a random orientation.

For incidence along the [110] axis direction, the broadness of $F_{[110]}\left(Q_{\text {out }}\right)$ reflects the distribution of their transverse energy in the crystal : the best channeled projectiles (about $50 \%$ of the incident beam), that have avoided close atomic collisions and have experienced only low electron densities in the crystal, have been able to keep all their electrons and are transmitted frozen in their initial charge state. The charge state group from 57 to $\sim 66$ reflects the transverse energy distribution of channeled projectiles : the higher $E_{\perp}$, the higher the electron density encountered and the higher the ionization probability. At last, projectiles of critical transverse energy, that are able - like projectiles in random conditions - to explore any region of the crystal, are able to reach very high charge states, up to 78. At first sight it could be thought that critical projectiles reach charge state equilibrium faster than in random conditions because they experience higher atomic densities. However we give also on Figure 3 the charge distribution $F_{\text {crit }}\left(Q_{\text {out }}\right)$ measured for a nearly critical incidence, i.e. for an incidence angle to [110] close to the critical angle $\Psi_{c}$ : these distributions, that include frozen or nearly frozen projectiles, possibly channeled along some minor planar direction, extend significantly beyond the -previously discussed- calculated distribution on the high charge state side. In particular He-like ions $\left(Q_{\text {out }}=80\right)$ are observed, that cannot be observed in random conditions with our target (and our counting statistics) and that could hardly be observed with a thicker 
one. The effect is still more visible on the last distribution $F_{2 E_{R}}\left(Q_{\text {out }}\right)$ given on figure 3, that corresponds to the projectiles at critical incidence that have lost more than twice the amount of energy $\overline{\Delta E}_{R}$ lost by random projectiles transmitted with the same charge state (see figure 5). This distribution (not normalized) shows clearly that projectiles that suffer a "higher than normal" energy loss, i.e. that spend much time close to [110] atomic strings, reach higher charge states than in random conditions, which implies a specific balance between electron loss and electron capture.

\subsubsection{Analysis using Monte-Carlo simulations.}

In figure 4 , we compare the aligned $F_{[110]}\left(Q_{\text {out }}\right)$ and critical $F_{\text {crit }}\left(Q_{\text {out }}\right)$ experimental distributions of figure 3 to Monte Carlo simulations. Small adjustments on the total cross sections, beam angular divergence and impact parameter dependence of MEC and NII were brought in order to improve the overall agreement. The beam angular divergence was described by a $2 \mathrm{D}$ gaussian distribution with a $1 \mathrm{D}$ standard deviation $u_{\text {beam }}=0.2$ $\operatorname{mrad}$ (to be compared to $\Psi_{c}=1.19 \mathrm{mrad}$ for bare $\mathrm{Pb}$ ions).

For ions with low $E_{\perp}, F_{[110]}\left(Q_{\text {out }}\right)$ is governed by NII in the disordered layers (in particular oxide) on both faces of the sample, and by EII in the crystal. The expected ratio between NII and EII cross sections is of the order of $10^{3}$. As our experiment is performed on a very thin crystal $(1.12 \mu \mathrm{m})$, the contribution of the disordered layers to ionization of low $E_{\perp}$ ions is thus of prime importance. The values of the EII cross sections introduced in the simulations in order to fit $F_{[110]}\left(Q_{\text {out }}\right)$ and in particular the fractions $F_{[110]}(56)$ and $F_{[110]}(57)$ depend heavily on the value chosen for the thickness $x_{d i s}$ of the disordered layers. Thus, in this experiment, we cannot access to precise values of $\sigma_{E I I}$. The fit of $F_{[110]}\left(Q_{\text {out }}\right)$ presented in figure 4 could be obtained for various couples of $\sigma_{E I I}(n=3)$ and $x_{d i s}$. Note that since the maximum energy transfer in free electronelectron collisions is $T_{e \max }=15.8 \mathrm{keV}$, lower than the binding energy $B_{n}$ for electrons on the $\mathrm{K}$ - and L-shells, the values of $\sigma_{E I I}$ for $n=1,2$ are negligible. Prediction on $\sigma_{E I I}$ in the frame of the BEB (Binary-encounter Bethe) model is provided in ref. [16] (equation 6). If we introduce the corresponding value in our simulations $\left(\sigma_{E I I}(n=3)=1.35 \times 10^{-22} \mathrm{~cm}^{2}\right)$, the fit of $F_{[110]}\left(Q_{\text {out }}\right)$ can only be obtained by assuming two disordered layer $\approx 120 \AA$ each, which appears surprisingly high.

The measured random distribution $F_{R}\left(Q_{\text {out }}\right)$ gives information on the ratio between NII and MEC cross sections, but also on the ratios between the rates of cascades $\tau_{\text {casc }}^{-1}(n)$ and those of all the other processes: $\tau_{N I I}^{-1}(n)=N_{A} \sigma_{N I I}(n) V_{i o n}, \tau_{M E C}^{-1}(n)=N_{A} \sigma_{M E C}(n) V_{i o n}$, $\tau_{\text {exc }}^{-1}(n)=N_{A} \sigma_{\text {exc }}(n) V_{\text {ion }}$, and to a much lesser extent $\tau_{E I I}^{-1}(n)=\rho \sigma_{E I I}(n) V_{i o n}\left(N_{A}=\right.$ 
$5.0 \times 10^{22} \mathrm{~cm}^{-3}$ is the atomic density and $\rho$ the electronic density encountered). The tail of the $F_{[110]}\left(Q_{\text {out }}\right)$ and the $F_{\text {crit }}\left(Q_{\text {out }}\right)$ distributions gives additional information on this balance and also on the impact parameter dependences $P_{N I I}(b, n)$ and $P_{M E C}(b, n)$ of NII and MEC for $n$ shell (see appendix A).

The experimental results are compatible with $P_{M E C}(b, n)$ narrower than $P_{N I I}(b, n)$. MEC feeds mainly $(n>2)$-shells, and the superdensity effect implies that ionization in outer shells for particles with high $E_{\perp}$ is more probable than in random conditions. One may then expect a possible dependence of the distribution charge at high $Q_{\text {out }}$ on the shape of $P_{M E C}(b, n>2)$ and $P_{N I I}(b, n>2)$ since the particle flux is strongly non uniform in channeling.

In order to estimate this dependence and more generally to clearly illustrate and quantify the difference of behavior of critical particles compared to that of random particles, we have considered the hypothetical case of ions moving on their entire pathway in a "critical medium". This is achieved by selecting particles with $E_{\perp}$ larger than a given threshold, by setting all the deflection angles to zero (rectilinear trajectories), by varying the shape of $P_{M E C}(b, n)$ or $P_{N I I}(b, n)$ and calculating the corresponding charge distributions $P_{\text {crit }}\left(Q_{\text {out }}\right)$. Such an hypothetical beam and medium correspond to a maximum superdensity effect. We have considered the fraction of $\approx 3.5 \%$ of the beam with $E_{\perp} / Q>70 e V$. The corresponding impact parameters $b_{\text {in }}$ at crystal entrance are $b_{\text {in }} \lesssim 0.25 \AA$.

We first studied the case where the $P_{N I I}(b, n)$ take the shapes of the $P_{M E C}(b, n)$ (impact parameter range smaller than $0.25 \AA$ ). At charge equilibrium, the superdensity effect results in a global increase of all the frequencies, except that of cascades (leading to an apparent decrease of the cascade frequency only). In the random situation, one has $\left(\bar{Q}_{\text {out }}=68.2\right)$ and the L-shell is nearly full (mean population 7.7$)$. Here, $F\left(Q_{\text {out }}\right)$ is found broader than $F_{R}\left(Q_{\text {out }}\right)$, the proportion of ions with high $Q_{\text {out }}$ is strongly enhanced $\left(\bar{Q}_{\text {out }}=72.3\right)$ and the mean population of the L-shell is 5.2. The fraction of ions with $Q_{\text {out }} \geqslant 73$, which is $1.4 \%$ for $F_{R}\left(Q_{\text {out }}\right)$, increases to $44 \%$.

Then, we performed simulations using the shape for $P_{M E C}(b, n)$ calculated from the CDW-EIS (continuum distorted wave eikonal initial state) approximation and $P_{N I I}(b, n)$ given by the core electronic densities. Let us recall (see appendix A) that the CDWEIS calculations predict a smaller extension for $P_{M E C}(b, n)$ than for $P_{N I I}(b, n)$. The simulations indicate that the fraction of ions emerging with $Q_{\text {out }} \geq 73$ reaches $55 \%$ and that $\bar{Q}_{\text {out }}=72.9$, illustrating the fact that superdensity effects are indeed somewhat sensitive to the relative spatial extensions of $P_{M E C}(b, n)$ and $P_{N I I}(b, n)$. However, the shift of the charge distribution with respect to the random case, which is about $\Delta Q=4$ and corresponds to a large modification of the tightly bound L-shell population, should be 
mainly attributed to the high collision rate. For real oscillating trajectories, the high $E_{\perp}$ ions spend a much smaller fraction of their path close to the rows than in the above ideal situation and are then far from equilibrium, resulting in a lower $\bar{Q}_{\text {out }}$. The experimental result $F_{\text {crit }}\left(Q_{\text {out }}\right)$ obtained by tilting the beam at critical incidence yields a fraction $14.2 \%$ of ions with $Q_{\text {out }} \geq 73$, much higher than the $1.4 \%$ random value, but indeed smaller than the $55 \%$ upper limit.

In conclusion, the anomalous high $Q_{\text {out }}$ events obtained experimentally in $F_{2 E_{R}}\left(Q_{\text {out }}\right)$ and $F_{\text {crit }}\left(Q_{\text {out }}\right)$ may be attributed to an effect of superdensity : for $P b^{56+}$ ions entering the $1.1 \mu \mathrm{m}$ crystal close to a dense [110] atomic row, the L- and M-shell ionization probabilities are much larger than in random conditions. The probability of electron capture by MEC in a collision with a Si atom is also enhanced, but the electrons are captured into outershells $(n \geq 4)[17]$ and are then likely to be rapidly lost in subsequent collisions with following Si atoms $(29 \mathrm{MeV} / \mathrm{u} \mathrm{Pb}$ ions pass along at least a few tens of atoms in a glancing collision with a [110] atomic row). These results show that critical channeling in a thin crystal could be used for producing high charge states that could not be obtained using ordinary stripping foils.

\subsection{Energy loss.}

\subsubsection{Experimental results}

Figure 5 represents the energy loss spectra $F_{[110]}(\Delta E)$ and $F_{R}(\Delta E)$ of the transmitted beam respectively for [110] alignment and for a random orientation, as measured by the high resolution magnetic spectrometer of the SPEG beam line. As for $F\left(Q_{\text {out }}\right)$, the broadness of the aligned energy loss spectrum $F_{[110]}(\Delta E)$ reflects the distribution in transverse energy of the beam. Energy losses up to $\approx 2.5$ times the random mean energy loss $\overline{\Delta E}_{R}$ are observed. The random spectrum $F_{R}(\Delta E)$ corresponds to ions emerging with $Q_{\text {out }}=68$, a value which is close to the mean value $\bar{Q}_{\text {out }}=68.5$ for the whole random beam. The losses $\Delta E$ are normalized to the mean random value $\overline{\Delta E}_{R}=13.5$ $\mathrm{MeV}$ for $Q_{\text {out }}=68 . F_{R}(\Delta E)$ is very broad (full width at half maximum FWHM $=1.6$ $\mathrm{MeV}$ ) when compared to the Bohr straggling [18] (see equation 7 in appendix A) with $\rho=Z_{t} N_{A}(\mathrm{FWHM}=0.69 \mathrm{MeV})$. This extra broadening obviously arises from the statistics of charge exchange processes (see [19] and references therein), that are not considered in the Bohr model. The mean values $\overline{\Delta E}_{R}\left(Q_{\text {out }}\right)$ of the measured random spectra are found to depend on $Q_{\text {out }}$, which us not surprising since, for the thin target used, charge state equilibrium is not reached. This dependence may be expressed by the empirical law $\Delta E_{R}\left(Q_{\text {out }}\right)=\left(6.8+\left(Q_{\text {out }}^{2}+Q_{\text {in }}^{2}\right) \times 8.6 \times 10^{-4}\right) \mathrm{MeV}$. 
The maximum value of $F_{[110]}\left(\Delta E / \overline{\Delta E}_{R}\right)$ corresponds to well channeled ions with $Q_{\text {out }}$ close to $Q_{i n}$. The tail of $F_{[110]}\left(\Delta E / \overline{\Delta E}_{R}\right)$ which extends up to more than $\Delta E=2 \times$ $\overline{\Delta E}_{\text {Random }}$ corresponds to high $E_{\perp}$ particles which experience close collisions with the atomic rows. This long tail is related to the variations of the energy transfers $T(b)$ as a function of the impact parameter to target nuclei. This experimental result demonstrates that, in the thin crystal used, statistical equilibrium is not reached and that "critical projectiles" experience in the average more collisions at small impact parameter than random ions.

\subsubsection{Monte-Carlo analysis}

The energy loss spectra calculated by Monte-Carlo for [110] alignment and random orientation are compared to the experimentally measured spectra in figure 5. The qualitative features of the experimental spectra are reproduced, in particular, the tail on the high loss side of $F_{[110]}\left(\Delta E / \overline{\Delta E}_{R}\right)$. However, strong discrepancies appear. The value $\Delta E_{\max }$ at the maximum of the calculated $F_{[110]}$ is significantly smaller than the measured one. The experimental value $\Delta E_{\max } / \Delta E_{R}=0.41$ (with $\Delta E_{R}=13.5 \mathrm{MeV}$ ) is surprisingly high since this maximum corresponds to well channeled ions with low $Q$. For ions with a given $Q$, the electron gas model applied to valence and core electrons predicts, for random geometry, that energy transfers to the valence gas, $\overline{\Delta E}_{\text {Rval }}$, represents $37.5 \%$ of the total loss $\overline{\Delta E}_{R}$. Using the Monte-Carlo code one finds that the mean quadratic charge state value $\bar{Q}_{R}$ in the target for a random beam is 63.6 . For the channeled ions with the most probable loss $\Delta E \approx \Delta E_{\max }$, the corresponding $\bar{Q}$ is very close to $Q_{i n}=56$. If channeled ions emerging with $\Delta E \approx \Delta E_{\max }$ would interact only with valence electrons and in a non local way, one should expect a ratio $\Delta E_{\max } / \Delta E_{R}=\left(Q_{\text {in }} / \bar{Q}_{R}\right)^{2} \overline{\Delta E}_{R v a l} / \overline{\Delta E}_{R}=0.29$, much smaller than the experimental value found (0.41). This shows that for well channeled ions, the energy loss to core electrons is important. The Monte-Carlo simulations yield $\Delta E_{\max } / \Delta E_{R}=0.30$, when using the LDA approximation, still markedly smaller than the experimental value. This indicates that the LDA approximation is not appropriate for describing the impact parameter dependence of the energy loss to the L-shell electrons of Si atoms at large impact parameter: interactions are not purely local and $T(b)$ should extend at larger distances than predicted by LDA.

The energy loss $\Delta E^{L}$ to core electrons for well channeled ions (i.e. at large impact parameter $b$ ) may be estimated by using a classical harmonic oscillator model [18][2]. The energy transfer to an $L$ electron at distance $b$ is approximately given by $T_{L}(b)=$ $R\left(b / b_{a d}\right) T^{\text {free }}(b)$, where $T^{\text {free }}(b)$ is the energy transfer to a free electron at distance $b$ and $R\left(b / b_{a d}\right)$ is a reduction factor which accounts for the adiabaticity of the interaction 
(here, $b_{a d} \simeq 4 \AA$ ). Integrating over the transverse space available for particles emerging with $\Delta E$ close to $\Delta E_{\max }\left(Q_{\text {out }}=56, E_{\perp}<15 \mathrm{eV}\right)$, one finds a mean value $R \simeq 0.5$ and $\Delta E^{L} \simeq 1.4 \mathrm{MeV}$. Adding up this energy loss to the Monte-Carlo calculated $\Delta E_{\max }^{L D A}$ in the LDA approximation yields $\Delta E_{\max }=5.5 \mathrm{MeV}=0.41 \Delta E_{R}$, in total agreement with the measured value.

In the Monte-Carlo calculations, using the Bohr straggling model (equation 7) for the valence gas and the LDA $T(b)$ law for core electrons leads to a broader random energy loss spectrum $(\mathrm{FWHM}=2.3 \mathrm{MeV})$ than experimentally measured $(1.6 \mathrm{MeV})$. As indicated in section 4.2.1, the experimental random spectrum is already broad, in connection with charge fluctuations. The latter are well taken into account in the simulations and the discrepancy between simulation and experiment arises from the fact that the LDA model strongly overestimates the fluctuations of energy transfers to core electrons. The very high calculated fluctuations may be analyzed analytically. Neglecting the contribution of the charge fluctuations, in random geometry, since the particle flux is uniform, the variance $\Omega_{\text {core }}^{2}$ associated to $\Delta E_{\text {core }}$ is given by

$$
\Omega_{\text {core }}^{2}=N_{A} z \int_{0}^{\infty} 2 \pi b d b T^{2}(b)
$$

and the corresponding to the mean value is:

$$
\overline{\Delta E}_{\text {core }}=N_{A} z \int_{0}^{\infty} 2 \pi b d b T(b)
$$

By numerical integration, one may calculate the relative fluctuations given by the ratio $r_{\text {core }}=\Omega_{\text {core }} / \Delta E_{\text {core }}$, which depends on the shape of the $T(b)$. The $r_{\text {core }}$ calculated from the LDA approximation is too high, demonstrating again that the shape of the $T(b)$ deduced from the LDA approximation is inappropriate. In fact, a broader distribution should give smaller fluctuations. This may be evidenced by considering for example an exponential shape $T(b)=A \exp \left(-b / b_{o}\right)$, where $A$ and $b_{o}$ are constant values (the latter gives the width of the distribution). One finds $\Omega / \overline{\Delta E}=\sqrt{2 / \pi} /\left(b_{o} \sqrt{N_{A} z}\right)$ i.e. the broader the distribution, the smaller the relative fluctuations.

In conclusion, for the ion considered, the experimental results obtained by measuring energy loss spectra in channeling and random geometry are sensitive to the impact parameter dependence $T(b)$ of the energy loss to core electrons and should deserve further investigation (see section 2.3). 


\section{Channeling experiment with $\mathrm{U}^{91+}$ ions.}

\subsection{Charge state distributions}

In this experiment with $20 \mathrm{MeV} / \mathrm{u} \mathrm{U}^{91+}$ ions, capture is the dominant process. The adiabaticity parameters $\eta_{n}=m_{e} V_{i o n}^{2} / 2 B_{n}$ (binding energies for $n<4, B_{1} \simeq 130 \mathrm{keV}$, $B_{2} \simeq 32.5 \mathrm{keV}, B_{3} \simeq 12 \mathrm{keV}$ etc.., $\left.\frac{1}{2} m_{e} V_{i o n}^{2}=10.9 \mathrm{keV}\right)$ are smaller than $1\left(\eta_{K}=0.084\right.$, $\left.\eta_{L}=0.34, \eta_{M}=0.91\right)$. The $\eta_{n}$ give qualitative information on the filling of the shells at charge state equilibrium: for $\eta_{n} \gg 1$ the $n$-shell is almost empty and conversely, for $\eta_{n} \ll 1$ the $n$-shell is almost full. In random conditions, the $\mathrm{K}$ - and L-shell are hence rapidly filled and the filling of the M-shell results from a balance between MEC, NII, cascades and excitation, and involves in particular $(n>3)$-shells.

In figure 6, we present charge state distributions obtained at GSI for various crystal orientations relative to the incident beam direction. In random conditions, the distribution (normalized to unity) is centered on a mean value of 73.6, in fair agreement with the value of 74.2 deduced from the semi-empirical formula of Leon et al. [14]. Here, contrary to the $\mathrm{Pb}^{56+}$ case, the crystal is thick enough for projectiles transmitted in random conditions to reach charge state equilibrium. When the beam is aligned with the [110] direction of the crystal, the associated normalized distribution is quite broad and dominated by the charge $90+(25 \%)$ and the frozen charge $91+(27 \%)$. These two fractions represent the best channeled projectiles, that encounter only low densities of quasi-free electrons. They may capture electrons only by REC, a process that is overwhelmed by MEC in random conditions (in fact as in the $\mathrm{Pb}^{56+}$ experiment, there is a small contribution of MEC in the thin amorphous surface layers). The decreasing charge states correspond to projectiles of increasing transverse energies : they may accede to regions of larger and larger electron densities and specially undergo closer and closer atomic collisions that increase both electron loss and capture probabilities. In particular the gaussian-like low charge tail is associated to projectiles of high transverse energy that experience atomic collisions down to zero impact parameter, just like projectiles in random conditions can do. One could then expect those projectiles to reproduce the random charge distribution. Instead, their charge states appear to be shifted on the higher charge state side by $\sim 3$ units. This effect becomes even more visible if one selects projectiles of high transverse energy : for this we used the property of secondary electron emission induced by projectiles in alignment conditions to increase with the transverse energy, as we have recently shown [4]. In figure 6 , we show the charge distribution for the fraction $(2 \%)$ of emergent ions that are associated with the largest multiplicity of forward emitted electrons (this choice was dictated by experimental constraints, i.e. the poor multiplicity resolution of our 
backward electron detector). In spite of the fact that the selection is not strict (due to the statistical nature of electron emission), one can see that the gaussian-like distribution yielded by critical projectiles is again shifted, by $\sim 2-3$ units, with respect to the distribution obtained in random conditions.

This superdensity effect goes far beyond the usual density effect [8] in solids. At $20 \mathrm{MeV} / \mathrm{u}$, critical uranium projectiles experience in part of their trajectory very high collision rates, and the mean time between two collisions is of the order of $10^{-17} \mathrm{~s}$, i.e. a time two orders of magnitude shorter than the time for electrons captured in a U outershell $(n \geq 4)$ to cascade down to the K- or the L-shell (typically $10^{-15} s$ ).

\subsection{X-ray spectra.}

Strong evidence for the superdensity effect is also given by the high energy resolution X-ray spectra obtained with a tightly collimated Ge detector (figure 7). We present here three spectra. The first one has been obtained in random conditions (figure 7- $a$ ) and the two others are energy spectra of X-rays detected in coincidence with ions of charges from 76 to 79 (figure 7-b) and with ions of charge 90 (figure 7-c), respectively, transmitted in [110] alignment conditions. The three spectra correspond to the same number of transmitted $U$ ions. The spectrum of figure $7-b$ is associated to transmitted ions that reached charge states also observable in random conditions and then may be considered as critical projectiles. The spectra of figs 7- $a$ and 7- $b$ are dominated by L- and K-line photons that are due to decay cascades following MEC events. The spectrum of figure 7-c is associated to channeled projectiles that have captured one electron, either by MEC or REC (they are not the very best channeled ones, that stay frozen in their initial charge state). This spectrum clearly shows the L- and K-REC peak positions. All these spectra have been discussed in some detail in [13], but what we want to emphasize here is the comparison between the two spectra due to random (figure 7-a) and critical (figure 7-b) projectiles.

First, REC essentially vanishes in random conditions : this happens because L- and K-shells are very rapidly filled by MEC, a process of much larger cross section than REC. The small amount of REC photons in figure 7-a tells us that the L- and K-shells are filled within $\sim 10^{-15} \mathrm{~s}$, that is much shorter than the dwell time of the projectile in the target $\left(\sim 210^{-14} \mathrm{~s}\right)$.

In contrast, L- and K-REC lines show up more visibly in figure $7-b$, in spite of the large probability for a MEC event to occur during the first glancing collision, the duration of which is also about $\sim 10^{-15} \mathrm{~s}$. We attribute this enhancement of REC to the high electron 
density encountered in atomic rows. However it could be partly due to the longer survival or K- and L-shell vacancies resulting from the superdensity effect.

The second point, that is more surely related to superdensity, deals with the comparison of L-lines shapes in spectra of figs 7- $a$ and 7-b, that exhibit a striking difference : in random conditions, for which the mean charge at emergence is 74 (the L-shell is full for $Q=82$ ), the sequential filling of the L-shell by MEC takes place relatively rapidly in the target. This process feeds L-lines with photons, the energies of which depend on the instantaneous charge of the projectile and then result in broad lines. Deeper in the target, individual L electrons may be lost by excitation/ionization in close collisions and the filling of these vacancies yields photons of much better defined energies that produce the two sharp peaks appearing in figure 6- $a$ over the broad base. These narrow components do not show up (or at least are very weak) in alignment conditions with critical projectiles (figure 6- $b$ ). The superdensity effect prevents part of the captured electrons from decaying to the L-shell; it results that filling the L-shell takes much more time than in random conditions, which attenuates strongly the narrow components of L-lines.

\subsection{Monte-Carlo analysis.}

\subsubsection{Fitting charge state distributions and determining the REC yield and associated electron density at ion site.}

We present in figure 8 the charge state distributions calculated by Monte-Carlo for [110] and random orientations. The calculated $F_{[110]}\left(Q_{\text {out }}\right)$ distribution reproduces the main features of the experimental one, in particular the fraction of frozen ions and the secondary maximum around $Q_{\text {out }}=77$. Only two parameters were varied in order to search for a good overall agreement: the beam angular divergence and the REC yields. A fair agreement is obtained by introducing a beam divergence represented by the sum of two $2 \mathrm{D}$ gaussian law $G, G_{\text {beam }}=0.2 \times G\left(u_{\text {beam } 1}\right)+0.8 \times G\left(u_{\text {beam } 2}\right)$, with $1 \mathrm{D}$ standard deviations $u_{\text {beam } 1}=0.25 \mathrm{mrad}$ and $u_{\text {beam } 2}=0.35 \mathrm{mrad}$ (the critical angle for channeling is $\Psi_{c}=1.42 \mathrm{mrad}$ ). Like for the $\mathrm{Pb}^{56+}$ experiment, the silicon crystal is covered by a thin amorphous layer on both faces. The observation of the charge state distribution and X-ray REC lines for a low multiplicity of electrons gives the mean number $N_{\text {layer }}^{M E C}$ of MEC in the entrance disordered layer. One finds $N_{\text {layer }}^{M E C} \approx 0.1$. The corresponding thickness of equivalent silicon is $\approx 4 \mathrm{~nm}$ (on both sides) and was included in the Monte-Carlo simulations. The fraction (here $27 \%$ ) of the frozen $U^{91+}$ is a measure of the REC yield. The mean electron density experienced by the ions emerging at $Q_{\text {out }}=90$ as determined by Monte-Carlo is $\bar{\rho}_{\text {vis }} \simeq 1.6 \times 10^{23} \mathrm{~cm}^{-3}$ (compared to $\left.\bar{\rho}_{\text {val }}=2 \times 10^{23} \mathrm{~cm}^{-3}\right)$. In order to 
reproduce the frozen fraction one has to increase the REC yield by a factor of $\approx 1.5$. This yield is equal to the product $\sigma_{R E C} \rho_{v i s} x_{o}$, where $x_{o}$ is the crystal thickness. Actually, the value of $\sigma_{R E C}$ for capture in $\mathrm{K}$ - and L-shell is given with a good precision by the Strobbe formula [20]. The thickness $x_{o}$ is known with a precision of $\approx 10 \%$ from the mean energy loss measured in random geometry. Thus, we may further analyze the factor of 1.5 that we apply in our simulations. A first reason for this factor may originate from the polarization of the electron gas, that increases the local electron density around the highly charged projectiles, with moderate velocity. We estimate this enhancement to be a factor of 1.2 to 1.6 (see E. Testa et. al., these proceedings). A second possible reason comes from the fact that since we limited MEC capture to $n \leq 5$ levels, we underestimate MEC probabilities at impact parameters $>0.3 \AA$. Indeed, capture to $n \geq 5$ occurs at large impact parameters and, in order to fit the experimental charge state distribution for high $Q_{\text {out }}$, the corresponding enhancement of the capture probability should be compensated for by an increase of the REC probability. This artefact has no consequences on the physics mainly discussed in this paper, i.e. charge exchange close to the atomic strings.

\subsubsection{Charge state distributions and superdensity effect.}

The calculated random distribution $F_{R}\left(Q_{\text {out }}\right)$ is in good agreement with the experimental one (nearly same mean value $\bar{Q}_{\text {out }}=73.6$ ). It was obtained without any change in the calculated cross sections (the modification of the calculated $\sigma_{E I I}$ has negligible influence on the results), indicating at least that the balance between electron capture and loss is well reproduced. The mean number of events for the $11.7 \mu \mathrm{m}$ target as calculated by Monte-Carlo are high: $N_{\text {capt }}=76, N_{\text {loss }}=58, N_{\text {exc }}=166$ and $N_{\text {casc }}=245$, indicating that a charge equilibrium is reached. Moreover, the K- and L-shells are filled and the mean population of the other shells are $\bar{N}_{3}=5.0, \bar{N}_{4}=1.35$ and $\bar{N}_{5}=2.1$. This means that whatever the rate $\tau_{N I I}^{-1}(n)$ of NII events is, ionization of excited states may occur. Hence, one may also analyze the density effect in random geometry as follows. It is essentially governed by the value of $\bar{N}_{4}+\bar{N}_{5}$ which in turns results from a balance between, on the one hand, excitation and capture in excited states and, on the other hand, cascading and ionization. Except for cascading, all the associated characteristic frequencies are proportional to $N_{A} V_{i o n}$. Hence, the equilibrium charge state distribution in a solid may depend strongly on the $\tau_{\text {casc }}(n)$ values even if $\tau_{\text {casc }}(n) \ll \tau_{N I I}(n)$. This may be easily evidenced using the Monte-Carlo code: if one increases by two orders of magnitude the probability of cascading per unit time, one obtains $\bar{Q}_{\text {out }}=69.6$ instead of $Q_{\text {out }}=73.6$, although in both cases one has $\tau_{\text {casc }}(n) \ll \tau_{N I I}(n)\left(N_{\text {casc }} \simeq(245 / 58) N_{\text {loss }}\right.$ for nominal $\tau_{\text {casc }}(n)$ values $)$.

For projectiles experiencing superdensities, the above discussion is in fact still valid, 
the only condition being that the cross sections are large enough so that over the duration $\tau_{\text {close }}$ of the close interaction of the ion with a row (plane) several charge exchange events may occur. In fact, changing from random to critical ions has similar effects than increasing the rate of all the events, but the cascading rate, resulting in a higher $\bar{Q}_{\text {out }}$ value. When the particle leaves the vicinity of the row (plane) the frequency of interaction is suddenly lowered, leading to a partial depopulation of the excited states towards more tightly bound states; however, the high superdensity $Q$ value reached during the glancing collisions is conserved.

\subsubsection{Analyzing the X-ray spectra}

The shape of the Balmer lines are connected to the evolution of the ions towards an equilibrium charge state, which may be studied using the Monte-Carlo code. For the random alignment, with the CDW-EIS NII and MEC cross sections one finds the following dependence of the mean $\bar{Q}_{\text {out }}$ value with the travelled path $x$ : $Q_{\text {out }}=79.3,75.05,74.0$, 73.7 and 73.6 respectively for $x=x_{o} / 6, x_{o} / 3, x_{o} / 2,2 x_{o} / 3$ and $x_{o}=11.7 \mu m$. Hence, over more than a half of their travelled path in the crystal, the ions are at charge equilibrium, and over a much greater fraction of their path, the K- and L-shells are filled, a result which is compatible with the shape of the measured Balmer lines for the random case.

For the channeled distribution, $\bar{Q}_{\text {out }}$ is not illustrative enough for our X-ray analysis, and one may study the evolution of the shape of $F_{[110]}\left(Q_{\text {out }}\right)$ with $x$. For a channeled beam, the trend towards charge state equilibrium is significantly slower than for a random beam. Since the equilibrium is not reached very rapidly for the random case, one may anticipate that it is not reached at all over the whole crystal thickness $x_{o}$ for the channeled beam. The trend towards equilibrium for low $E_{\perp}$ ions is extremely low (nearly no MEC nor NII, low EII and REC cross sections). The question is more intricate for high $E_{\perp}$ particles which is the relevant group of particles if one is concerned with the analysis of the shape of the Balmer lines. When comparing $F_{[110]}(Q)$ at various values of depth $x$ for ions with $Q_{\text {out }} \leqslant 80$ one finds by Monte-Carlo a proportion of $17.2 \%$ for $x=x_{o}$ and $4.5 \%$ for $x=x_{o} / 2$ evidencing a strong non equilibrium situation for the high $E_{\perp}$ ions in the tail of the $F_{[110]}\left(Q_{\text {out }}\right)$ distribution. When considering the group of particles with $E_{\perp}>70$ $\mathrm{eV}\left(3.5 \%\right.$ of the beam), one finds the following mean population $N_{e}(n)$ of the shells: for $x=x_{o} / 2$, one has respectively $N_{e}(n=1$ to 5$)=1.93,4.75,1.65,0.23,0.31$ (mean charge state 83.1) and for $x=x_{o}$ one finds $1.98,6.2,2.75,0.40$ and 0.55 (mean charge state 80.1). Thus, not only the mean charge state, but also the population of each electronic level for $n>1$ varies significantly with $x$ all along the ion path. Moreover, the charge state distribution of these high $E_{\perp}$ ions is extremely broad, extending form 91 to 65 . 
These simulations confirm then the fact that for critical particles the filling of the L-shell is never completed in the target, leading to the broad Balmer lines in figure 7- $b$.

\section{Conclusion.}

We have used heavy ion channeling in thin crystals to show that charge exchange of fast projectiles in atomic rows does not result only in an increase of loss and capture probabilities, but present specific aspects due to the high collision rate they experience. This superdensity effect, that prevents captured electrons from cascading down to projectile innershells and leaves ions in higher charge states than in random conditions, could e.g. be used to efficiently strip heavy ions in accelerator technology. The authors are very grateful to P.N. Abufager and R. D. Rivarola for providing CDW-EIS calculations. This work was partially supported by the $\mathrm{IN}_{2} \mathrm{P}_{3}$-GSI collaboration agreement.

\section{Appendix A: Monte-Carlo simulations}

\subsection{Trajectories}

In the Monte-Carlo description (binary collisions), only two parameters are necessary for calculating trajectories for given incidence conditions: the ion-atom potential $V(R)$ and the thermal vibrations. For a given particle entering the crystal, the trajectory is calculated independently from the charge state fluctuations. This means that $V(R)$ is calculated once for all and is stationary along the trajectory. If the charge of the ions exhibits large variations, the potential is calculated for a mean representative charge. The thermal vibrations are represented through the independent oscillator model, i.e. the atomic displacements are given by a gaussian law with a variance $\rho^{2}$ calculated from the Debye theory and an experimentally determined Debye temperature $\theta_{D}=450 \mathrm{~K}$ (the $1 \mathrm{D}$ standard deviation for thermal vibration is $u_{1}=0.077 \AA$ and the corresponding $2 \mathrm{D}$ value is $\rho=u_{1} \sqrt{2}=0.109 \AA$ ). Correlations of thermal vibrations are not considered in our simulations. They slightly affect channeling and can be simply accounted for, in the frame of a Markov chain description, by introducing a single correlation coefficient $r$ between the displacements of neighbouring atoms. However, $r$ should be adjusted to the phonon spectrum of a Si crystal, and this has not been already achieved.

For each crossing of the successive (100) planes of the silicon crystal, the closest vibrating atom is searched for and the angular deflection of the trajectory is calculated. 
All the positions and impact parameters of the successive binary collisions are memorized for a given ion and a given target thickness. From these data, energy loss and charge exchange are calculated.

For calculating $V(R)$, we use the Moliere [21] analytical approximation of the ThomasFermi screening function $\varphi_{T F}(R / a)$. In our experimental situation (highly stripped heavy ions and low $Z_{t}$ target), the screening radius $a_{T F}$ was chosen to be that of silicon $a_{S i}$ i.e. the ion is assumed to be a point charge at the scale of silicon orbitals, with mean value $\bar{Q}_{\text {ion. }}$. Then

$$
V(R)=\frac{\bar{Q}_{i o n} Z_{t} e^{2}}{R} \varphi_{T F}\left(R / a_{S i}\right)
$$

This choice differs from that proposed in ref [22] for the rather light ions used in Ion

Beam Analysis, $V(R)=\frac{Z_{i o n} Z_{t} e^{2}}{R} \varphi_{T F}(R / a)$. In this latter case, the screening radius $a$ depends on $Q_{\text {ion }}$ and describes both target atom and ion screening.

The random geometry may be obtained by introducing a large angular divergence for the beam and choosing an incident direction far form any axial or planar orientation. In fact, one may use a much simpler procedure: for each collision, the 2D impact parameter $\mathbf{b}_{\perp}$ is randomly chosen (uniform density) on a disk of appropriate area $\pi R_{o}^{2}$ (the probability density for $b$ is $P(b)=2 b / R_{o}$ for $\left.0<b<R_{o}\right)$.

\subsection{Energy loss}

In order to study the energy loss processes of ions as a function of their transverse energy, an impact parameter approach is needed.

\subsubsection{Energy loss to valence electrons}

The energy loss to the valence gas may be estimated by using two extreme hypotheses:

i) assuming that the ions interact with a uniform free electron gas with density $\bar{\rho}_{\text {val }}=$ $4 N_{A}=2 . \times 10^{23} \mathrm{~cm}^{-3}$ (where $N_{A}$ is the atomic density of silicon):

$$
-\left(\frac{d E}{d z}\right)_{v a l}=\frac{4 \pi Q_{i o n}^{2} e^{4} \bar{\rho}_{v a l}}{m_{e} V_{i o n}^{2}} \ln \left(\frac{2 m_{e} V_{i o n}^{2}}{\hbar \omega_{p}\left(\bar{\rho}_{v a l}\right)}\right)
$$

where $V_{i o n}$ is the ion velocity, $\hbar \omega_{p}\left(\bar{\rho}_{v a l}\right)=16.6 \mathrm{eV}$ is the plasmon energy associated to the electron gas, and $m_{e}$ the electron mass. The Bohr parameter "kappa" [18], defined as the ratio between the collision diameter (closest distance of approach) and the wavelength (divided by $2 \pi$ ) associated to the particle is given by $\kappa=2 Z_{\text {ion }} V_{B} / V_{\text {ion }}$ where $V_{B}=c / 137$ is the Bohr velocity. For $29 \mathrm{MeV} / \mathrm{u} \mathrm{Pb}, \kappa \simeq 4.8$ is larger than 1, pointing for a semiclassical description of the interactions. By analogy with the Bohr oscillator approach 
[18], for $\kappa>1$ the argument of the logarithm in (6) should be divided by $\kappa$, leading to a $\approx 20 \%$ decrease of the energy loss rate.

ii) assuming that an ion interacts with a free electron gas of density given by the local valence density $\left(\bar{\rho}_{\text {val }}\right.$ is replaced by $\rho_{v a l}\left(\mathbf{r}_{\perp}\right)$ in $\left.(6)\right)$. The density $\rho_{v a l}\left(\mathbf{r}_{\perp}\right)$ is calculated by averaging along the [110] direction the $3 \mathrm{D}$ valence density $\rho_{\text {val }}(\mathbf{r})$ calculated in ref. [23] using a pseudo-potential approach. $\rho_{v a l}\left(\mathbf{r}_{\perp}\right)$ is represented in fig $1-b$. In the center of the channel, $\rho$ is noticeably smaller than $\bar{\rho}_{v a l}$ leading in this approximation to smaller energy losses.

Experimental measurements [24], [25] show that for the ions considered here, hypothesis $i$ ) is much more realistic than hypothesis ii). This may be easily understood since, for the ion considered here, a pure Coulomb interaction with a medium energy transfer $T \simeq 100 \mathrm{eV}$ corresponds to rather large impact parameters $(b \simeq 0.7 \AA$ for $29 \mathrm{MeV} / \mathrm{u} \mathrm{Pb})$; moreover, the adiabatic cutoff $b_{a d}=V_{i o n} / \omega_{p}$ is very large $\left(b_{a d} \simeq 29 \AA\right.$, much larger than the interatomic distances) and low energy transfers associated to the collective excitation of the electron gas are nearly purely non-local.

\subsubsection{Energy loss to core electrons}

We need to introduce an impact parameter description for the energy loss to the core electrons. At the present stage of the code, we have used a well known semiclassical approach, namely the local density approximation (LDA). The ion is assumed to be a point charge at the scale of silicon core orbitals. In the LDA model, the atom is considered as a superposition of electron gases with various electronic densities $\rho_{\text {core }}(\mathbf{r})$. For a given impact parameter $b$, the energy transfer $T$ to core target electrons is calculated along the trajectory using equation (6) with the local density $\rho_{\text {core }}(\mathbf{r})$. This gives the energy transfer $T(b)$ for a given impact parameter with respect to the atomic nucleus. The use of the LDA approximation is of course highly questionable for the ions used in our experiments, that may involve excitation and ionization of L-shell Si electrons at rather large impact parameters (see section 2.3 and discussion in section 4.2.2).

In the LDA approximation, the energy straggling corresponding to energy loss to core electrons is directly given by the fluctuations of $T(b)$ corresponding to the fluctuations of $b$ from collision to collision. For the straggling of the electron loss to the valence gas, we used the simple Bohr expression [18]

$$
\Omega^{2} / z=4 \pi Z_{p}^{2} e^{4} \rho
$$


with, in the present case, $\rho=\bar{\rho}_{\text {val }}$.

In fact, there is another origin in energy straggling which is important in the experiment described in this paper. It arises from the fact that charge exchange processes induce charge fluctuations and hence energy loss fluctuations [19]. The Monte-Carlo code takes into account this effect since at each collision, the value of $Q_{i o n}$ in equation (6) is modified according to the charge exchange events.

\subsection{Charge exchange}

In what follows, we give information on the various cross sections and recombination probabilities used in our simulations. Let us recall that only shells corresponding to $n \leq 5$ were considered.

a) NII cross sections $\sigma_{N I I}$ were calculated [26] using the CDW-EIS approximation. The energy transfers are high and, using a classical point of view, correspond to impact parameters $b_{e}$ (between the Si nuclei and the electron of the ion) much smaller than the ion orbital extension. For a given ion electron in shell $n$, the variations with $b$ of the probability $P_{N I I}(b, n)$ of inducing a NII event for an impact parameter $b$ (between Si atoms and projectile ion nuclei) reflect hence the $n$-shell electronic orbital extension of the ion. $P_{N I I}(b, n)$ was then estimated from the ion $n$-shell electronic densities. $P_{N I I}(b, n)$ is normalized by $\int 2 \pi b d b P_{N I I}(b, n)=\sigma_{N I I}(n)$.

b) MEC cross sections $\sigma_{M E C}$ are calculated using the CDW-EIS approximations [27]. This theoretical approach gives the impact parameter probability $P_{M E C}(b, n)$ for MEC into a given shell $n$. Since MEC is a three-body process in which the energy and momentum conservations are obtained by means of the target atom recoil, the range of $P_{M E C}(b, n)$ is in our case smaller than that of NII for $n>2$. Capture in high $n$-shells plays a major role (large range, high cross sections) in charge exchange processes. As an illustration, the figure 9- $a$ gives $P_{M E C}(b, n)$ for $20 \mathrm{MeV} / \mathrm{u} \mathrm{U}^{91+}$ ions on silicon. These curves should be compared to the radial extension of the orbitals of $\mathrm{U}^{91+}$ of figure 9- $b$ : for a given electronic shell $n$, the range of $P_{M E C}(b, n)$ is typically twice smaller than that of $P_{N I I}(b, n)$.

c) Recombination probabilities per unit time $P_{\text {casc }}\left(n \rightarrow n^{\prime}\right)$ for transitions from a shell $n$ to a shell $n^{\prime}$ were taken from the calculations of Omidvar [15] for H-like ions. These probabilities scale as $Z_{p}^{4}$. For $M$ to $L$ transitions, Auger processes were included. The mean life time $\tau_{n}$ for an electron in an excited state in shell $n$ is given by $\tau_{n}^{-1}=$ $\sum_{n^{\prime}<n} P_{\text {casc }}\left(n \rightarrow n^{\prime}\right)$. 
d) REC cross sections were taken from Bethe and Salpeter [20] (calculation in the dipole approximation). REC is a local process, i.e. the probability to induce a REC event is proportional to the electron density $\rho_{e}\left(\mathbf{r}_{\perp}\right)$ at the ion site.

e) EII cross sections $\sigma_{E I I}(n)$ were evaluated from the binary encounter Bethe (BEB) model for tightly bound shells [16].

From all these data, one may calculate the probability $P_{i}$ to induce a given process (indexed by $i$ ) over a distance corresponding to the crossing of two consecutive (100) planes, taking into account the actual electronic population $N_{e}(n)$ on each ion shell, for a given impact parameter $b$ and position $\mathbf{r}_{\perp}$ in the channel. For each (100) plane crossing, the occurrence of each of the various $i$ processes are randomly chosen according to the $P_{i}$. Then the $P_{i}$ are recalculated to take into account a possible variation of $N_{e}(n)$. At large $b$, the $P_{i}$ are small and the probability for no event is large. On the contrary, for a collision at very small $b$, the MEC and NII $P_{i}$ values may be very high, leading to possible multiple events in the same collision. 


\section{References}

[1] S. Andriamonje, R. Anne, N. V. de Castro Faria, M. Chevallier, C. Cohen, J. Dural, M. J. Gaillard, R. Genre, M. Hage-Ali, R. Kirsch, A. L'Hoir, B. Farizon-Mazuy, J. Mory, J. Moulin, J. C. Poizat, Y. Quéré, J. Remillieux, D. Schmaus, and M. Toulemonde, Phys. Rev. Lett. 63 (1989) 1930.

[2] J.U. Andersen, J. Chevallier, G.C. Ball, W.G. Davies, J.S. Forster, J.S. Geiger, J.A. Davies, H. Geissel and E.P. Kanter, Phys. Rev. A 54 (1996) 624.

[3] H. F. Krause and S. Datz, Advances in atomic, molecular and optical physics vol 37, p. 139 (ed. B. Bederson and H. Walther (Academic Press, New York), 1996).

[4] F. Barrué, M. Chevallier, D. Dauvergne, R. Kirsch, J.-C. Poizat, C. Ray, L. Adoui, A. Cassimi, H. Rothard, M. Toulemonde, C. Cohen, A. L'Hoir, D. Vernhet, C. Demonchy, L. Giot, W. Mittig, S. Pita, P. Roussel-Chomaz, and A. Billebaud, Phys. Rev. A 70 (2004) 032902".

[5] T. A. Tombrello, Nucl. Instrum. and Methods 102 (1995) 312.

[6] I. Vickridge, A. L'Hoir, J. Gyulai, C. Cohen and F. Abel, Europhys. Lett. 13 (1990) 635.

[7] K. Komaki, T. Azuma, T. Ito, Y. Takabayashi, Y. Yamazaki, M. Sano, M. Torikoshi, A. Kitagawa, E. Takada and T. Murakami, Nucl. Instrum. and Methods B 146 (1998) 19.

[8] H. D. Betz and L. Grodzins, Phys. Rev. Lett. 25 (1970) 211.

[9] D.S. Gemmell, Rev. Mod. Phys. 46 (1974) 129.

[10] J. Lindhard, Mat. Fys. Medd. Dan. Vid. Selsk. 34, No 14 (1965).

[11] H. Ascolani and N.R. Arista, Phys. Rev. A 33 (1986) 2352.

[12] P. Sigmund and A. Schinner, Nucl. Instr. Methods B 195 (2002) 64.

[13] D. Dauvergne, A. Bräuning-Demian, F. Bosch, H. Bräuning, M. Chevallier, C. Cohen, A. Gumberidze, A. L'Hoir, R. Kirsch, C. Kozhuharov, D. Liesen, P.H. Mokler, J.C. Poizat, C. Ray, Th. Stölker, M. Tarisien, E. Testa, S. Toleikis and M. Toulemonde, Nucl. Instr. Methods B 205 (2003) 773.

[14] A. Leon, S. Melki, D. Lisfi, J.P. Grandin, P. Jardin, M.G. Suraud and A. Cassimi, At. Data Nucl. Data Tables, 69 (1998) 217. 
[15] K. Omidvar, Atomic Data and Nuclear Data Tables 28 (1983) 1.

[16] J.P. Santos, F. Parente and Y-K Kim, J. Phys. B 36 (2003) 4211.

[17] Dz. Belkic, R. Gayet and A. Salin, Comput. Phys. Commun. 32 (1984) 385.

[18] N. Bohr, K.Mat. Fys. Medd. Dan. Vid. Selsk., 27, No 15 (1948).

[19] P. Sigmund, Nucl. Instr. Methods B 69(1992) 113.

[20] H. A. Bethe and E. E. Salpeter, Quantum Mechanics of One and Two-Electron Atoms (Academic Press, New-York, 1957).

[21] Z. Moliere, Z. Naturforschung A2 (1947) 133.

[22] G. Amsel, G. Battistig and A. L'Hoir, Nucl. Instr. Methods B 201 (2003) 325.

[23] J.R. Chelikovsky and M.L. Cohen, Phys. Rev. B 14 (1976) 556.

[24] A. L'Hoir, S. Andriamonje, R. Anne, N.V. De Castro Faria, M. Chevallier, C. Cohen, J. Dural, M.J. Gaillard, R. Genre, M. Hage-Ali, R. Kirsch, B. Farizon-Mazuy, J. Mory, J. Moulin, J.C. Poizat, Y. Quéré, J. Remillieux, D. Schmaus and M. Toulemonde, Nucl.Instr. and Meth. B 48 (1990) 145.

[25] D. Dauvergne, C. Scheidenberger, A. L'Hoir, J. U. Andersen, S. Andriamonje, C. Böckstiegel, M. Chevallier, C. Cohen, N. Cue, S. Czajkowski, J. S. Forster, H. Geissel, H. Irnich, T. Kandler, R. Kirsch, A. Magel, P. H. Mokler, G. Münzenberg, F. Nickel, Yu. L. Pivovarov, J-C. Poizat, M. F. Politis, J. Remillieux, D. Schmaus, Th. Stöhlker, T. Suzuki, and M. Toulemonde, Phys. Rev. A 59 (1999) 2813.

[26] P.D. Fainstein, V.H. Ponce and R.D. Rivarola, J. Phys. B. 21 (1988) 287.

[27] P.N. Abufager, A.E. Martinez, R.D. Rivarola and P.D. Fainstein, J. Phys. B 37 (2004) 817. 

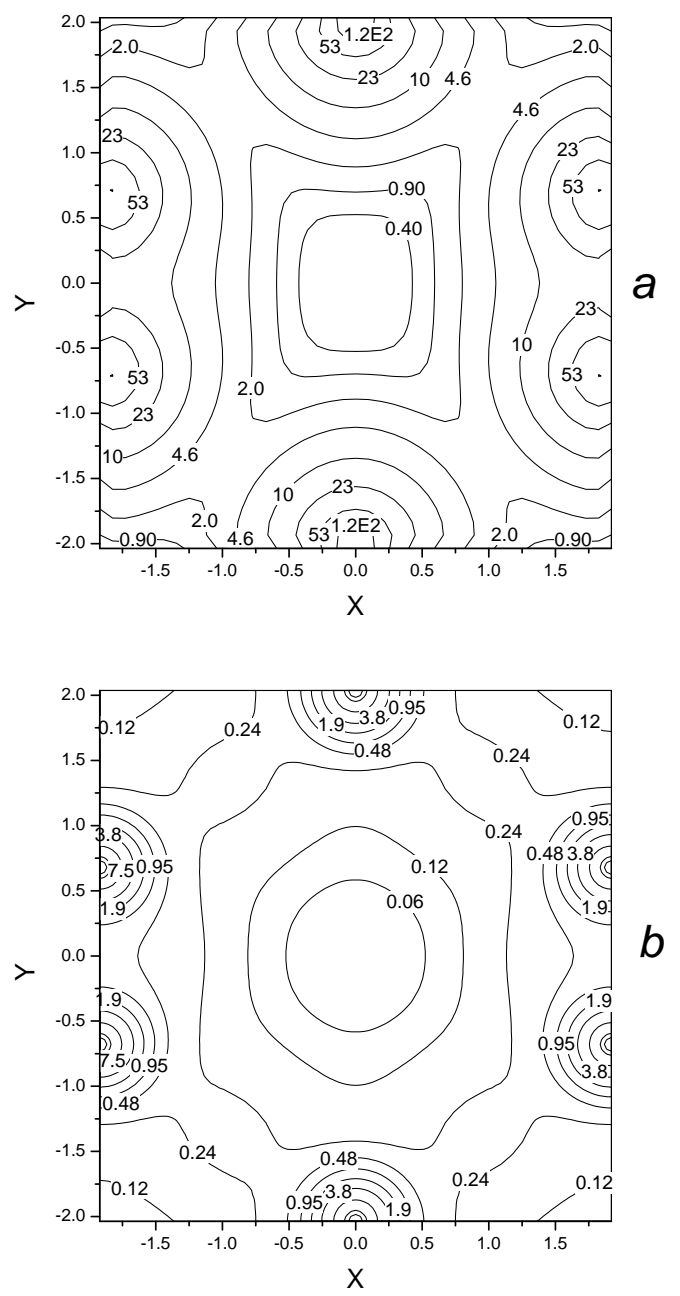

Figure 1: a) Potential in $\mathrm{Si}$ [110] channel for a unit charge, averaged along the [110] direction (labelled in $\mathrm{eV}$ ). b) Electronic density averaged along the [110] direction (labelled in electrons per cubic angström) 

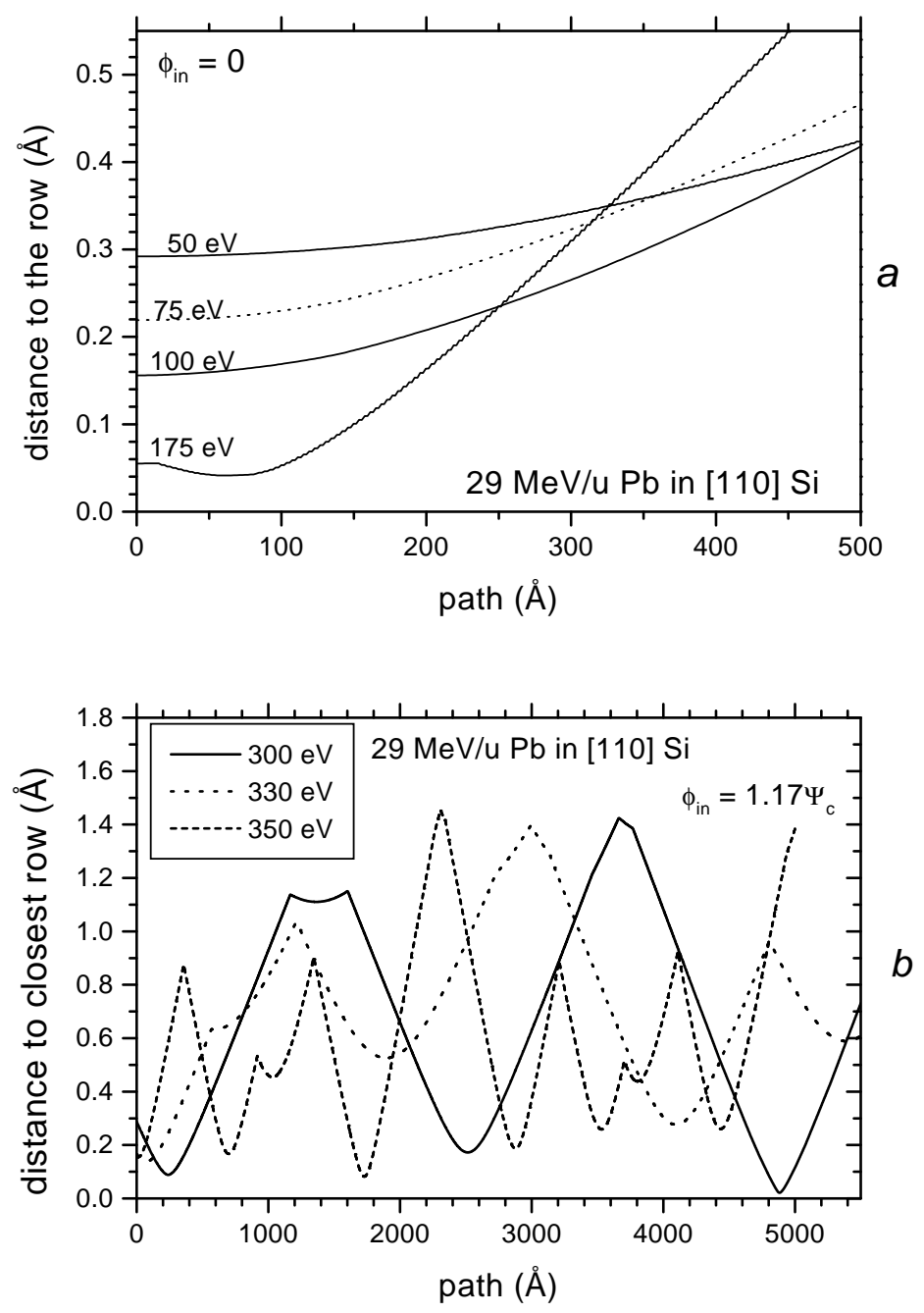

Figure 2: Trajectories of $20 \mathrm{MeV} / \mathrm{u} \mathrm{Pb}^{56+}$ along [110] silicon atomic rows. a) Ion trajectories for various transverse energies (beam aligned with [110] axis). b) Ion trajectories for a tilted beam and various transverse energies. 


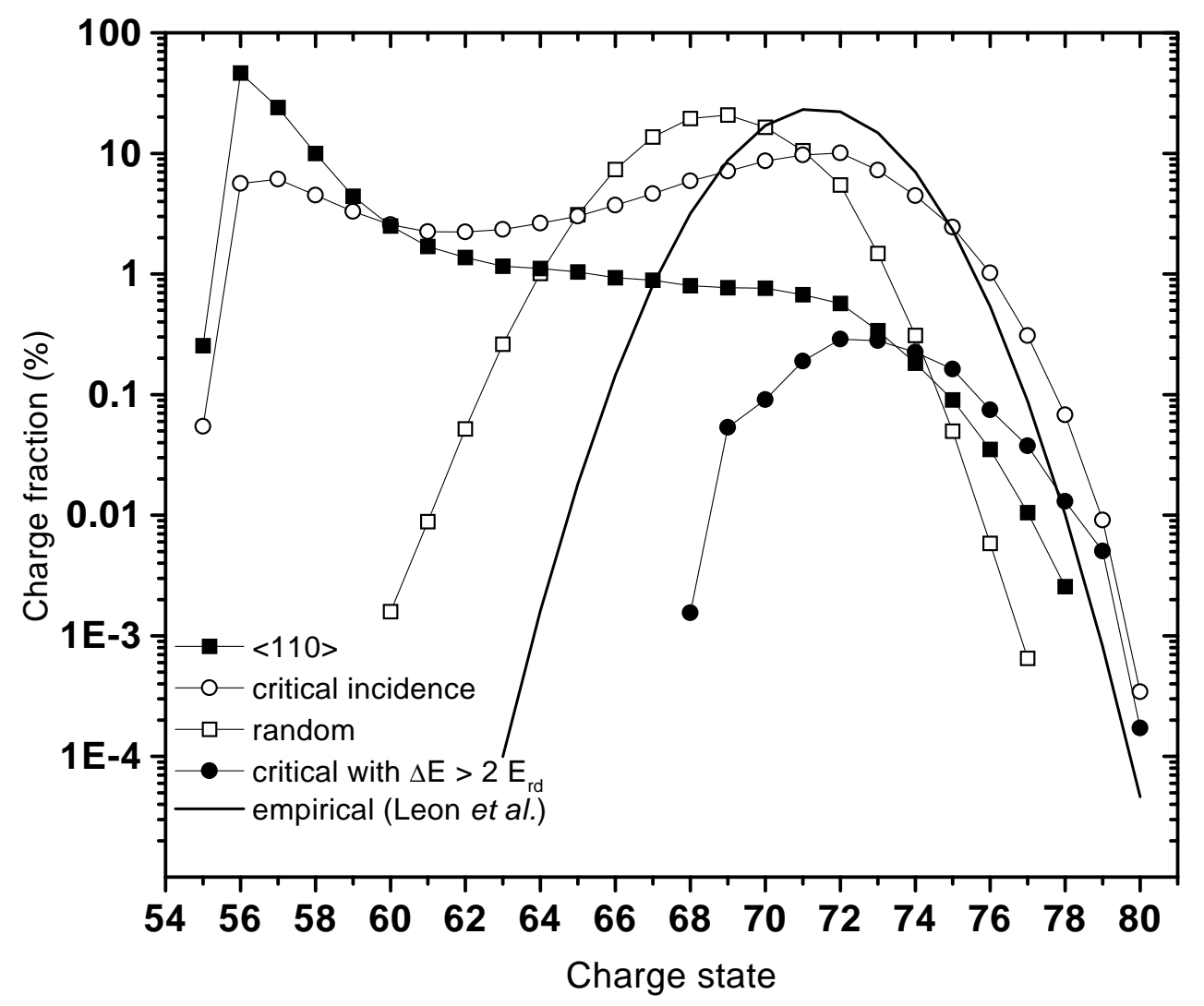

Figure 3: Charge state distributions for $29 \mathrm{MeV} / \mathrm{u} \mathrm{Pb}^{56+}$ incident ions after transmission through a $1.1 \mu \mathrm{m}$ silicon crystal : $F_{R}\left(Q_{\text {out }}\right)$ distribution for random incidence ( $\square$ ); $F_{[110]}\left(Q_{\text {out }}\right)$ distribution for [110] alignment $(\mathbf{\square}) ; F_{\text {crit }}\left(Q_{\text {out }}\right)$ distribution for critical incidence to [110] (o); $F_{2 E_{R}}\left(Q_{\text {out }}\right)$ distribution for the same conditions, with an energy loss equal to twice the random value $(\bullet)$. The solid curve is the distribution calculated from Leon et al. [14]. All distributions are normalized to $100 \%$, except $F_{2 E_{R}}\left(Q_{o u t}\right)$, which is normalized to the corresponding fraction. 

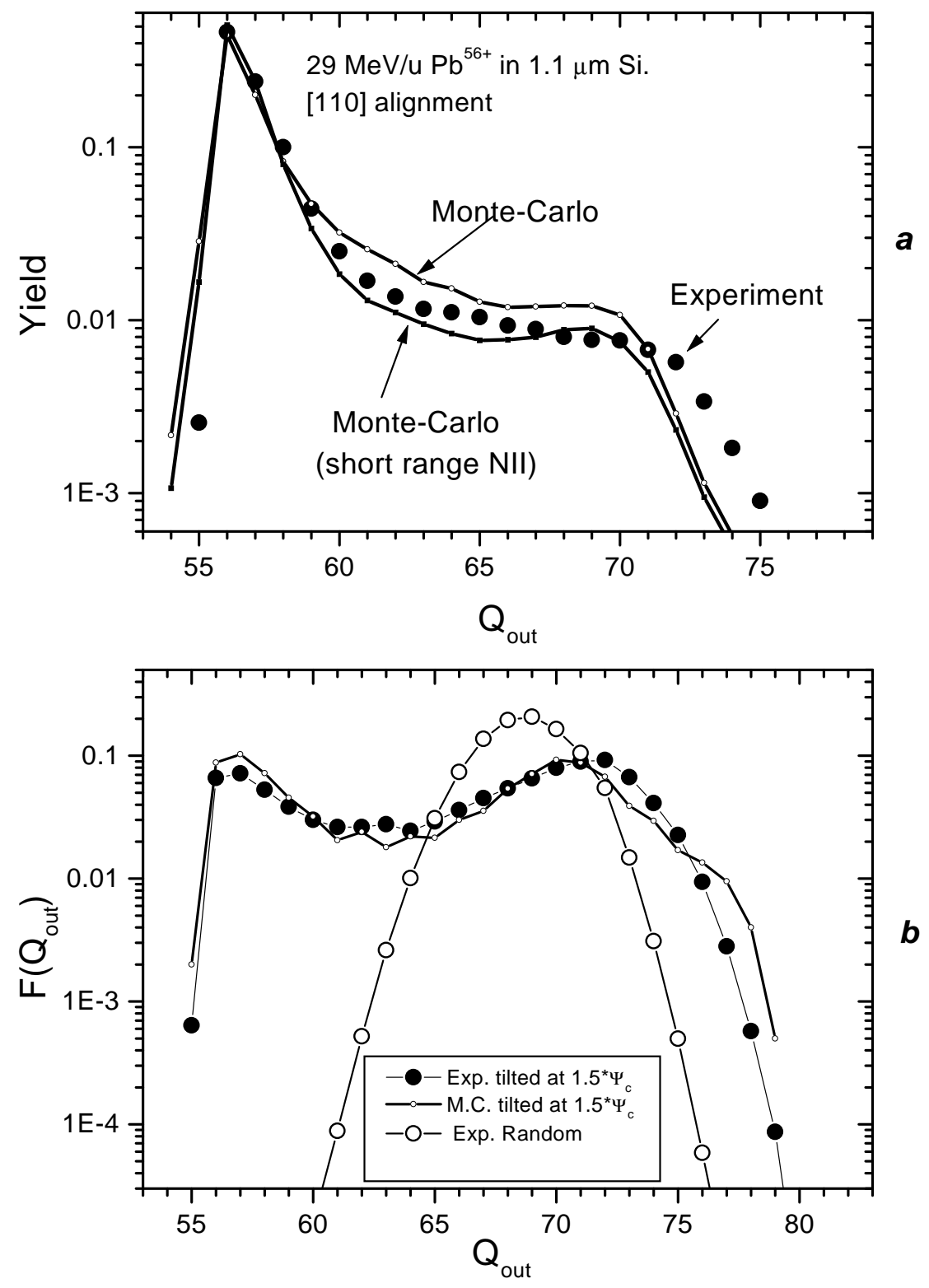

Figure 4: Measured charge state distributions for $29 \mathrm{MeV} / \mathrm{u} \mathrm{Pb}^{56+}$ ions channeled in a $1.1 \mu \mathrm{m}$ thick silicon crystal and corresponding Monte-Carlo calculated distributions. ) [110] alignment for two different impact parameter distributions for NII. ) For a tilted angle $1.5 \times \Psi_{c}$; the Omidvar [15] probabilities for cascading per unit times (see appendix A) were divided by a factor 5 . The distribution corresponding to a random orientation is also represented. 


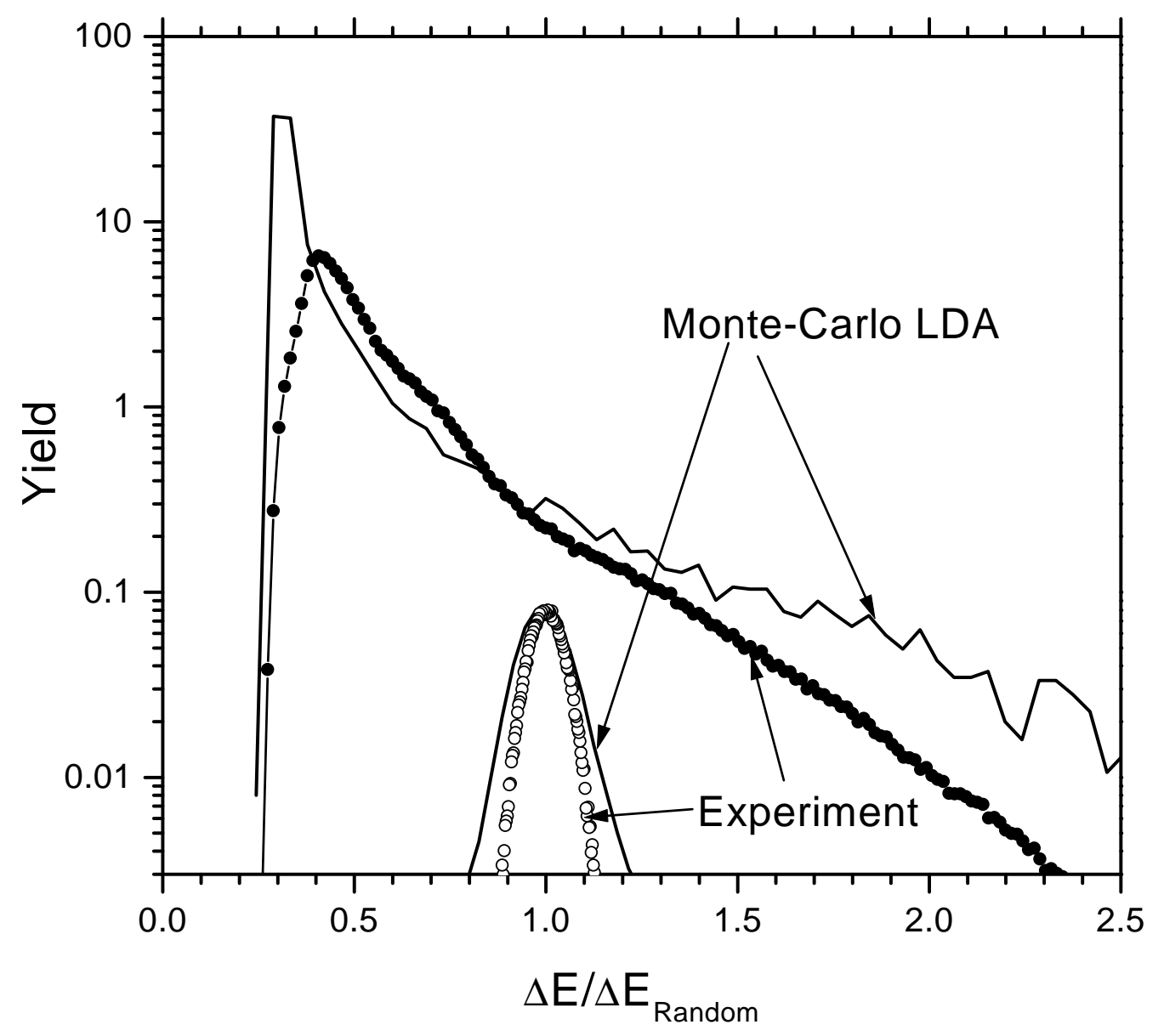

Figure 5: Energy loss spectra for $29 \mathrm{MeV} / \mathrm{u} \mathrm{Pb}^{56+}$ ions channeled along the [110] axis of a $1.1 \mu \mathrm{m}$ thick silicon crystal and for random geometry. Energy loss is normalized to the mean random energy loss for $Q_{\text {out }}=68$. Black and open circles: experimental spectra, respectively for aligned and random geometries. Solid lines: Spectra calculated by MonteCarlo. 


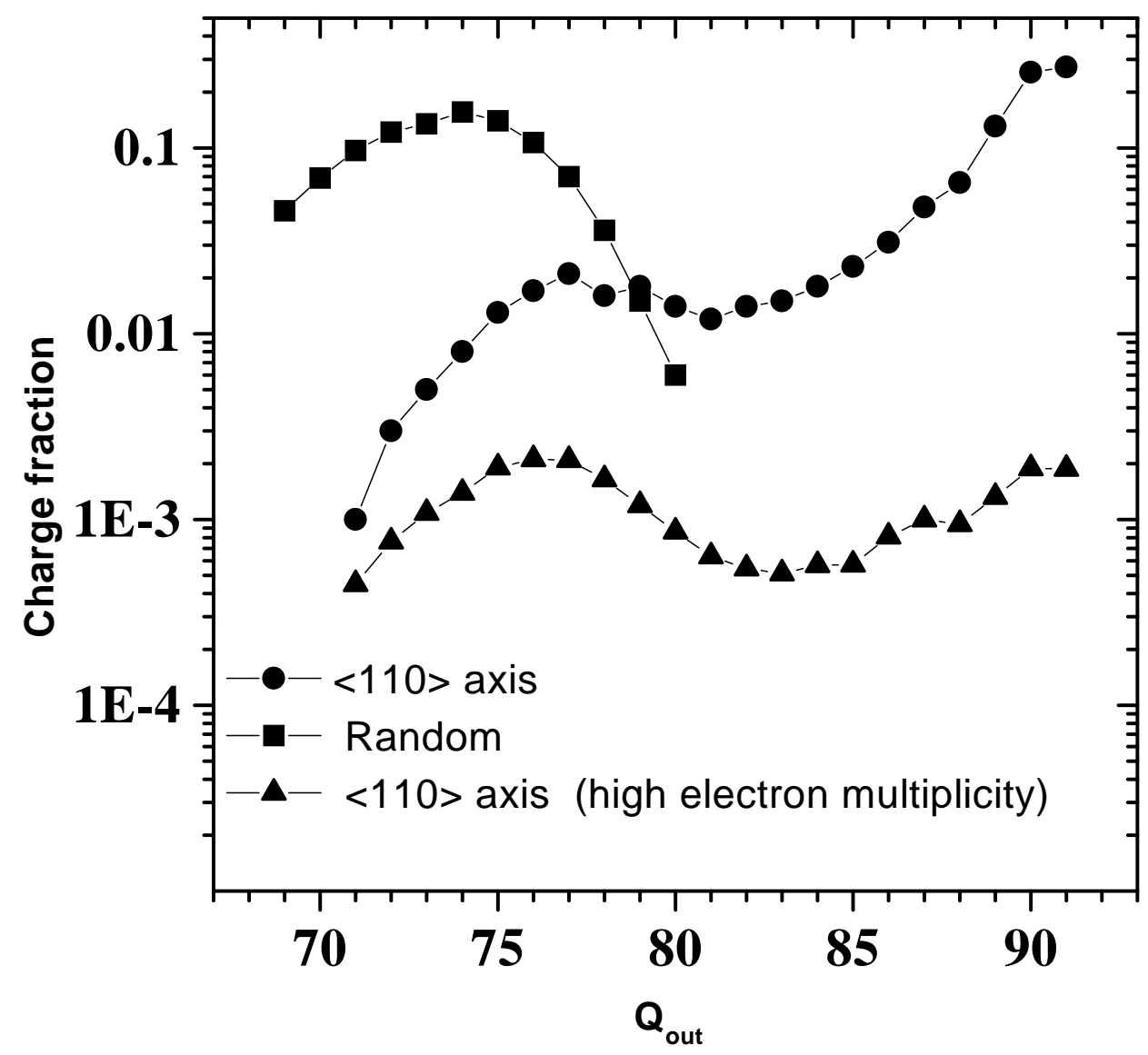

Figure 6: Charge distributions for $20 \mathrm{MeV} / u$ incident $U^{91+}$ ions after transmission through a $11.7 \mu m$ silicon crystal : random incidence $(\mathbf{\square}),[110]$ alignment $(\bullet),($ these two distributions are normalized to 1); [110] alignment and high electron multiplicity (ム) (see text); this last distribution is normalized to 0.02 which is the corresponding ion fraction. 


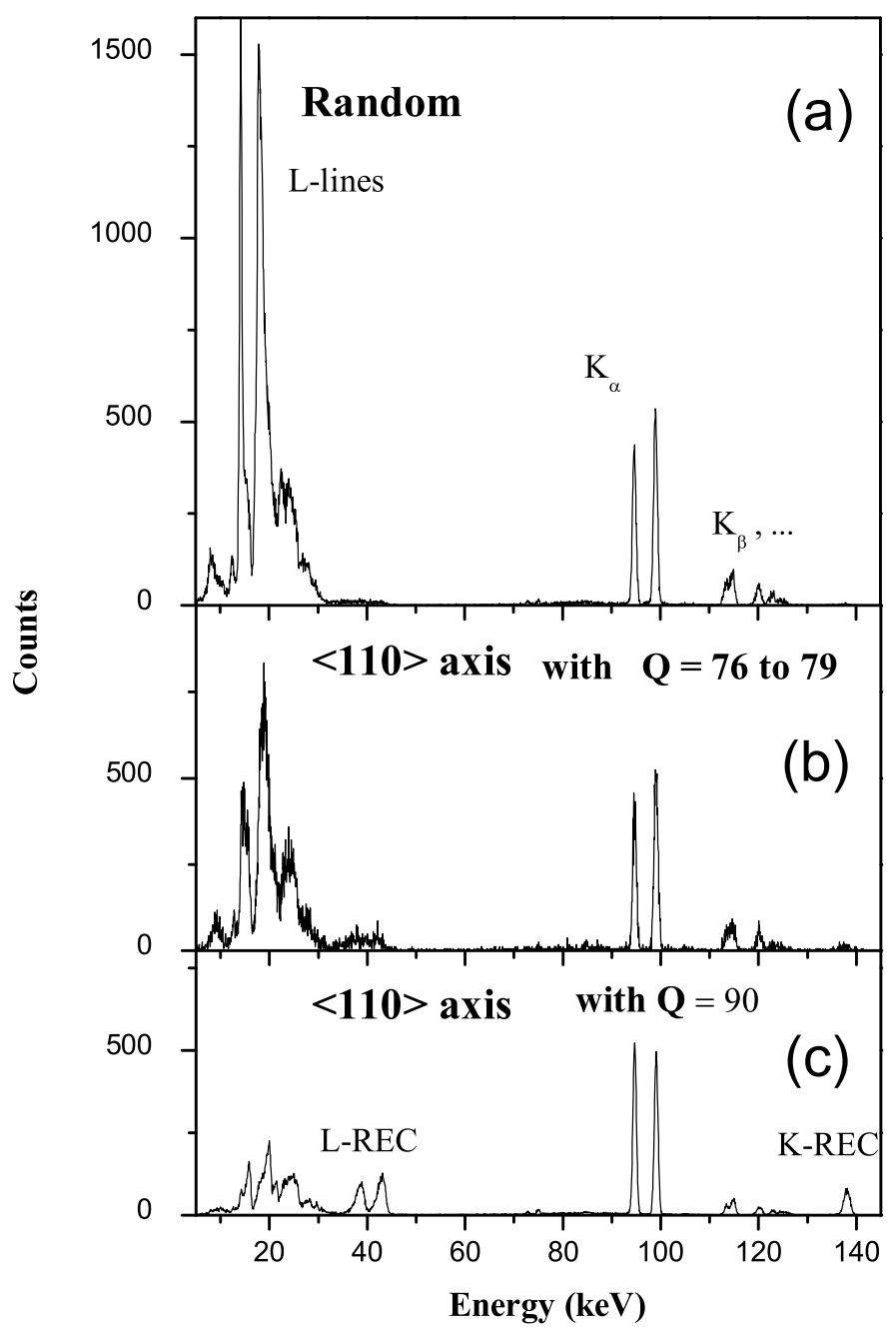

Figure 7: $20 \mathrm{MeV} / \mathrm{u} \mathrm{U}^{91+}$ ions incident on a $11.7 \mu \mathrm{m}$ Si crystal. X-ray recorded at $90^{\circ}$ to the beam : for random conditions (a), for [110] alignment and ions transmitted with charge states 76 to 79 (b), and with charge 90 (c). The spectra are normalized to the same number of transmitted ions with the given selection. 


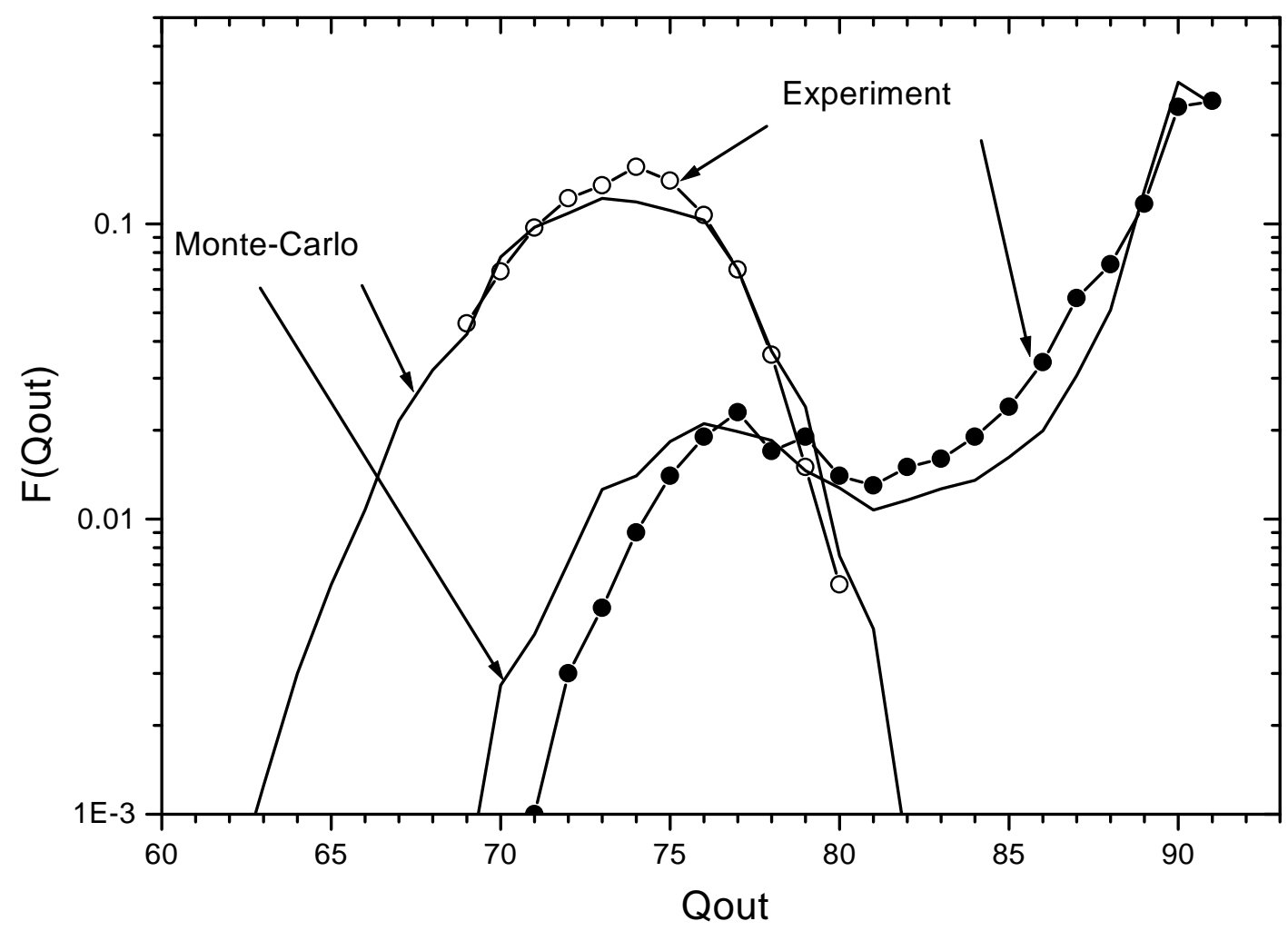

Figure 8: $20 \mathrm{MeV} / \mathrm{u} \mathrm{U}^{91+}$ ions incident on a $11.7 \mu \mathrm{m}$ Si crystal. Charge distributions for [110] axial (full circles) and random (open circles) geometries, and associated Monte-Carlo calculated curves. 


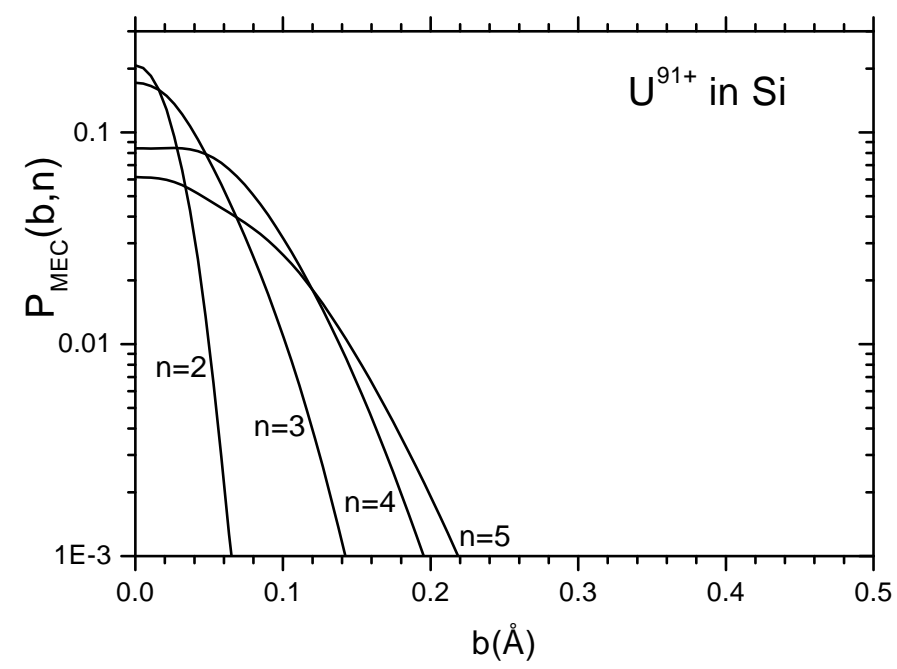

(a)

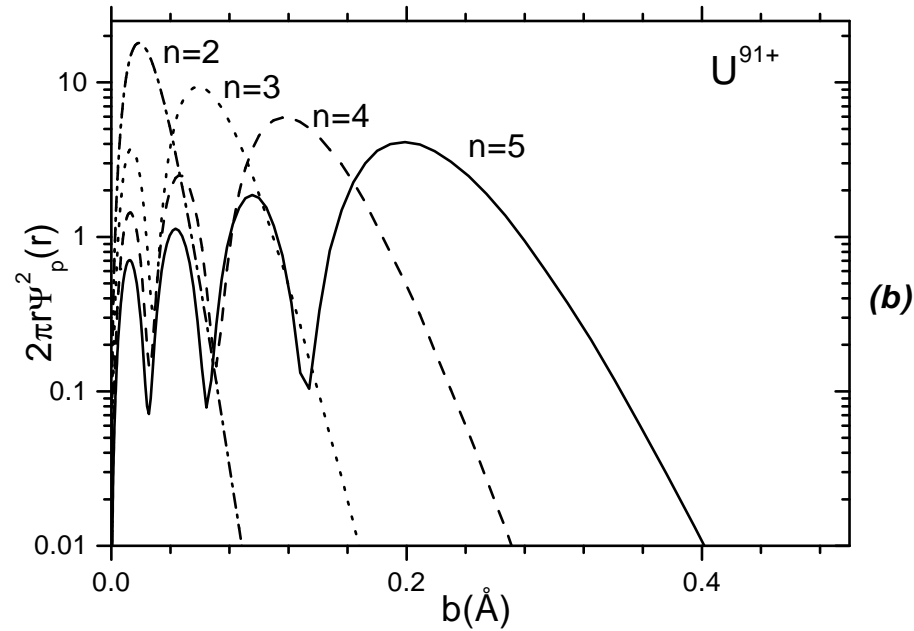

Figure 9: Impact parameter dependence of non radiative capture (MEC) and ionization by target nuclei (NII) for uranium in silicon. a) CDW-EIS calculated $P_{M E C}(b, n)$. b) Some orbitals of H-like uranium used to estimate $P_{N I I}(b, n)$ (for clarity, only $p$ orbitals are represented in the figure, indexed by $n=2$ to 5 ). 